\title{
Animierte thematische Karten zur Schweizer Bevölkerungsstatistik
}

\section{Einführung}

In allen raumbezogenen Wissenschaften - wie in nahezu allen gesellschaftlichen Bereichen auch - hat die explosionsartige Entwicklung und Ausbreitung moderner Kommunikations- und Informationstechnologien zu einer bislang ungekannten quantitativen wie qualitativen Zunahme von Informationen aller Art geführt. Allerdings ist eine direkte Verarbeitung der permanent anwachsenden Geodatenbestände nur noch im Einzelfall möglich. Daher werden immer mehr Massendaten, z. B. solche der Geostatistik, für die spätere Aufbereitung in Datenbanken und Informationssystemen gespeichert. Zugleich wächst aber auch die Kluft zwischen dem immer unüberschaubareren Informationsdargebot und einer effizienten Extraktion und sinnvollen Nutzung der benötigten Daten.

Zur Überwindung dieses ebenso gravierenden wie grundlegenden Problems werden neuartige Konzepte der Ordnung und Nutzung der Massendaten benötigt (z. B. POSTMAN 1995). Hierbei kommt der graphischen Informationspräsentation oder «Visualisierung» als einer effektiven Form der Kompression und Nutzung komplexer Massendaten eine Schlüsselrolle zu, wie jüngere Untersuchungen zur Nutzung von Massendaten (McCORMICK et al. 1987) verdeutlichen. Visualisierung zielt auf die «Sichtbarmachung» von Daten mittels Computergraphik und summiert somit ein ganzes Methodenbündel graphikbezogener Datenpräsentation (u. a. MacEACHREN/TAYLOR 1994, HEARNSHAW/UNWIN 1994). Abgesehen von ihrer informationstechnischen Komponente stellt die Visualisierung raumbezogener Daten aber kein völlig neues Verarbeitungs- und Nutzungskonzept dar. In den geographischen Disziplinen verfügt z. B. die Kartographie über eine lange Tradition der graphischen Veranschaulichung räumlicher Daten. Ebenso ist die Visualisierung statistischer Daten durch die nationalen statistischen Institutionen seit langem üblich.

Die dynamische Entwicklung der Informationstechnologie hat jedoch nicht nur die Möglichkeiten der graphischen Datenverarbeitung signifikant verbessert. Sie erlaubt auch die kombinierte digitale Verarbeitung moderner Datentypen wie Animation, Video und Ton mit den klassischen Datentypen Text, Graphik und Bild. Dieses Konzept der integrierten Informationsverarbeitung ist unter dem Begriff «Multimedia» allgemein bekannt und seit Mitte der neunziger Jahre auch in der
Kartographie verbreitet. Bezüglich des fachgerechten Einsatzes multimedialer Verfahren in der kartenbasierten elektronischen Informationsdiffusion besteht weiterhin erheblicher Forschungsbedarf. Das kartographische Nutzungspotential dieser multimedialen Techniken war Gegenstand eines anwendungsorientierten F+E-Projektes zur Visualisierung raumzeitlicher Massenstatistik, das 1994 bis 1995 in Zusammenarbeit mit dem Bundesamt für Statistik (BFS, Bern) durchgeführt wurde. ' Im nachfolgenden Beitrag werden einige konzeptionelle Grundlagen dieses Projektes dargelegt und ausgewählte dynamisch-interaktive Visualisierungen der Bevölkerungsstatistik diskutiert.

\section{Visualisierungskonzepte raumzeitlicher Dynamik}

Methodisch beruht die Visualisierung raumbezogener Daten in digitalen Karten - wie bei ihren konventionellen Pendants - auf den Grundsätzen der thematischen Kartographie. Technisch wird - im Unterschied zu herkömmlichen Karten - die Verarbeitung und Präsentation komplexer Rauminformationen durch Einsatz der Computergraphik realisiert (TAYLOR 1991a, MacEACHREN/ MONMONIER 1992, MacEACHREN 1994). Überwiegend hat sich die Visualisierung von Geodaten - z. B. mittels Desktop Mapping - bislang auf die optimale Modellierung statischer Zustandskarten gerichtet (ASCHE/ HERRMANN 1993). Form und Inhalt dieser gedruckten Karten können vom Nutzer nicht verändert werden. Durch Nutzung multimedialer Techniken und Instrumente erweitert sich die geographische Visualisierung aber auch auf die multidimensionale Modellierung raumzeitlicher $\mathrm{Zu}$ stände und Prozesse in dynamischen Karten. Dabei sind Form und Inhalt dieser virtuellen, flüchtigen Karten für den interaktiven, nutzergesteuerten Gebrauch auf Computergraphiksystemen zu modifizieren (DiBIASE et al. 1992, TAYLOR 1991a, 1994, MONMONIER 1992).

Christian Herrmann, Prof. Dr., FH Karlsruhe - Hochschule für Technik, FB Geoinformationswesen, Studiengang Kartographie, Moltkestraße 30, D-76012 Karlsruhe; christian.herrmann@fh-karlsruhe.de;

Hartmut Asche, Prof. Dr., Universität Potsdam, Institut für Geographie, Abt. Geoinformatik, Postfach 601553 , D-14415 Potsdam; gislab@rz.uni-potsdam.de. 


\subsection{Konventionelle Raum-Zeit-Synthesen}

Herkömmliche thematische Karten gelten als kartographische Realweltmodelle, die raumzeitliche Informationen in chorographischer und nichtchronologischer Form abbilden. Die Dynamik raumzeitlicher Entwicklungen läßt sich mittels derartiger Zustandskarten nicht adäquat und lediglich indirekt wiedergeben, was die ganzheitliche Erfassung und Auswertung raumzeitlicher Abläufe durch den Kartennutzer merklich beeinträchtigt. Als methodologische Basis der Modellierung statischer thematischer Karten kann das System der graphischen Variablen nach BERTIN (1974) angesehen werden, das die adäquate Visualisierung von Zeit und Bewegung nur unzureichend berücksichtigt. Die adäquate kartographische Präsentation der Raumdimensionen Zeit und Bewegung wurde folglich zu den ungelösten Fragen der thematischen Kartographie gerechnet (WITT 1970) und erst in jüngster Zeit - unter dem Einfluß der Computertechnik - stärker thematisiert (u. a. MacEACHREN 1994 a, b).

Ein verbreitetes konventionelles Verfahren, mit statischen kartographischen Mitteln raumzeitliche Dynamik wiederzugeben, ist die Erzeugung thematischer Kartenfolgen (Abb. 1). Hierbei bilden die Einzelkarten einer Folge unterschiedliche Zustände desselben raumzeitlichen Prozesses in statischen Momentaufnahmen ab. Die Prozeßhaftigkeit der Raumdaten wird durch eine Serie aufeinanderfolgender Standbilder visualisiert, in denen die raumzeitlichen Veränderungen jeweils auf der Zeitachse eingefroren sind (RASE 1974). So wurde im Bundesamt für Statistik (BFS) raumbezogene Massenstatistik dynamischer Prozesse wie z.B. der Bevölkerungsentwicklung bislang ausschließlich in statischen Zeitpunktkarten visualisiert, u.a. für das Statistische Jahrbuch der Schweiz (SJB). Die raumzeitliche Dynamik des Gesamtprozesses vermag der Kartennutzer lediglich indirekt durch einen visuellen Vergleich der Einzelkarten der Kartenfolge zu erfassen, indem er die Einzelkarten gedanklich zu einer dynamischen Vorstellungskarte (Mental map) synthetisiert. Allerdings stößt diese ohnehin komplexe Modellbildung rasch an Grenzen, wenn die raumzeitliche Synthese einer größeren Zahl von Karten einer Kartenreihe gefordert ist. Um gleichwohl die Informationsübermittlung raumzeitlicher Dynamik durch eine fachgerecht modellierte Kar-

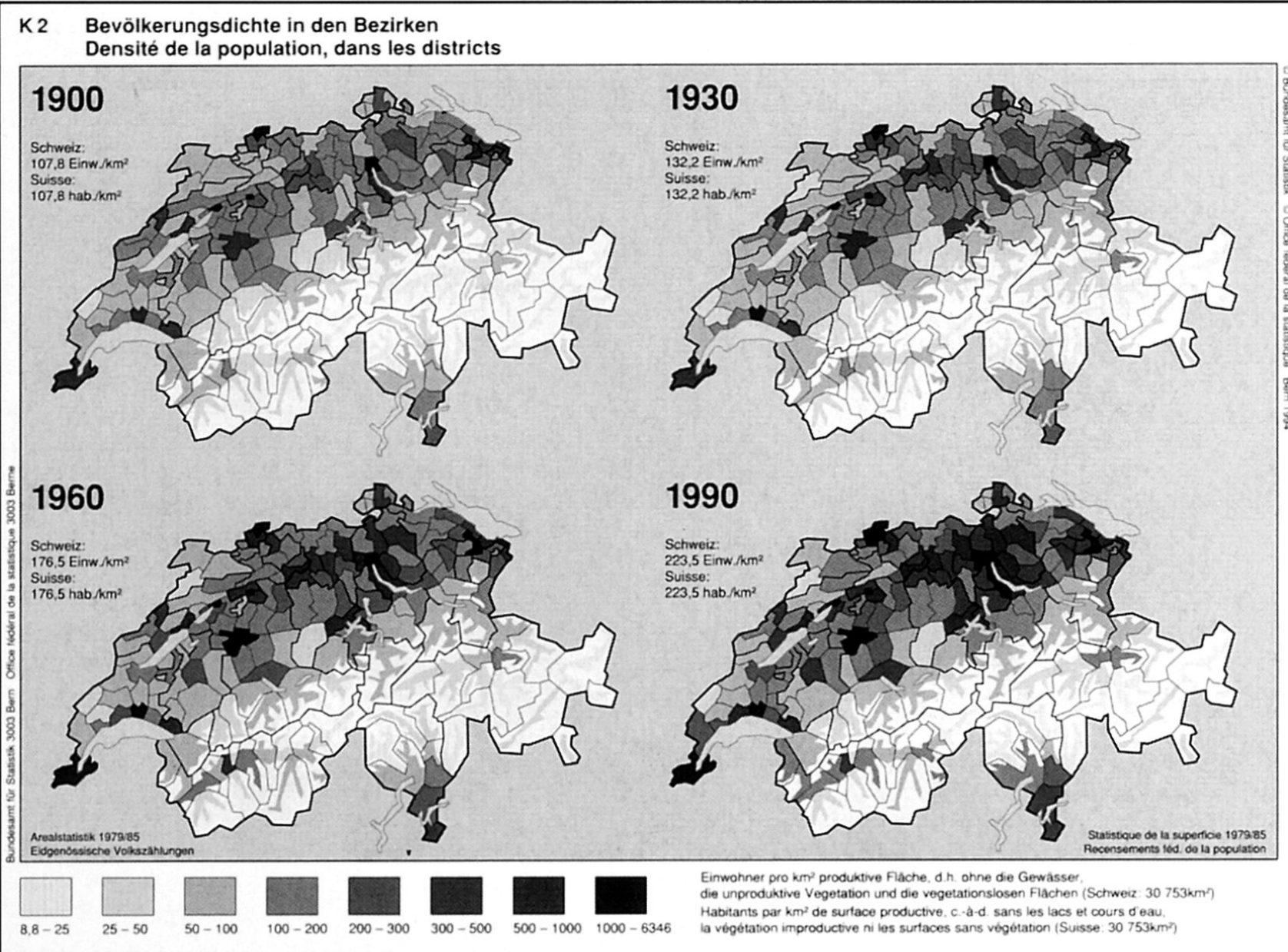

Abb. 1: Kartenfolge Bevölkerungsdichte: Bevölkerungsdichte in den Bezirken 1900, 1930, 1960, 1990 
tengraphik sicherzustellen, kann raumzeitliche Dynamik alternativ in einer Kartensynthese wiedergegeben werden (Abb. 2). Zeit und Bewegung werden in einer Kartensynthese hauptsächlich durch Pfeilsignaturen und Schriftzusätze dargestellt. Solch komplexe RaumZeit-Synthesen entsprechen einer generalisierten graphischen Zusammenfassung aller Zustandskarten. Auch diese stark generalisierte Bilanzdarstellung raumzeitlicher Prozesse erlaubt lediglich indirekte Rückschlüsse auf die jeweilige Prozeßdynamik. Vertiefte Einsichten in Details des raumzeitlichen Prozeßablaufs werden hierdurch erschwert. Sie können allein durch einen visuellen Kartenvergleich der Bilanzkarte mit u. U. verfügbaren Zustandskarten erreicht werden.

\subsection{Animierte Raum-Zeit-Karten}

Einen geeigneten methodischen Ansatz zur adäquaten Visualisierung raumzeitlicher Dynamik bietet die bereits vor 100 Jahren entwickelte kinematographische Technik. Dazu wird die Bewegung eines oder mehrerer Objekte, in 16 bis 30 Einzel- bzw. Standbilder pro Sekunde zerlegt, fortlaufend aufgenommen. Aufgrund der
Trägheit des menschlichen Auges wird die schnelle Wiedergabe der Standbilder als dynamische Bildsequenz, als Bewegtbild, empfunden und begrifflich als Bewegungsanimation charakterisiert. Der konventionelle Zeichentrickfilm kann als klassisches Medium der Bewegtbildgraphik gelten, in dem die grundlegenden Animationstechniken entwickelt wurden. ${ }^{2}$ Auf ihnen basiert die rechnergestützte Erzeugung von Bewegtbildern, die sogenannte Computeranimation, bei der Programme zur Erstellung kompletter Animationen aus digital erzeugten Einzelgrafiken eingesetzt werden. Computeranimationen werden nach Aufzeichnung im Regelfall auf Video oder am Computerbildschirm ausgegeben, seltener simultan zur Erzeugung in «Echtzeit» am Monitor angezeigt.

Der Einsatz rechnergestützter Verfahren zur Erzeugung von Animationen begann bereits in den fünfziger Jahren, ihre effiziente Anwendung wurde allerdings erst durch die Entwicklung leistungsstarker Rechner- und Programmsysteme möglich. Seither hat es nicht an Modellierungsversuchen gefehlt (z.B. TOBLER 1970, MOELleRING 1980), mit Mitteln der Computergraphik zu einer sachgemäßeren Visualisierung dynamischer

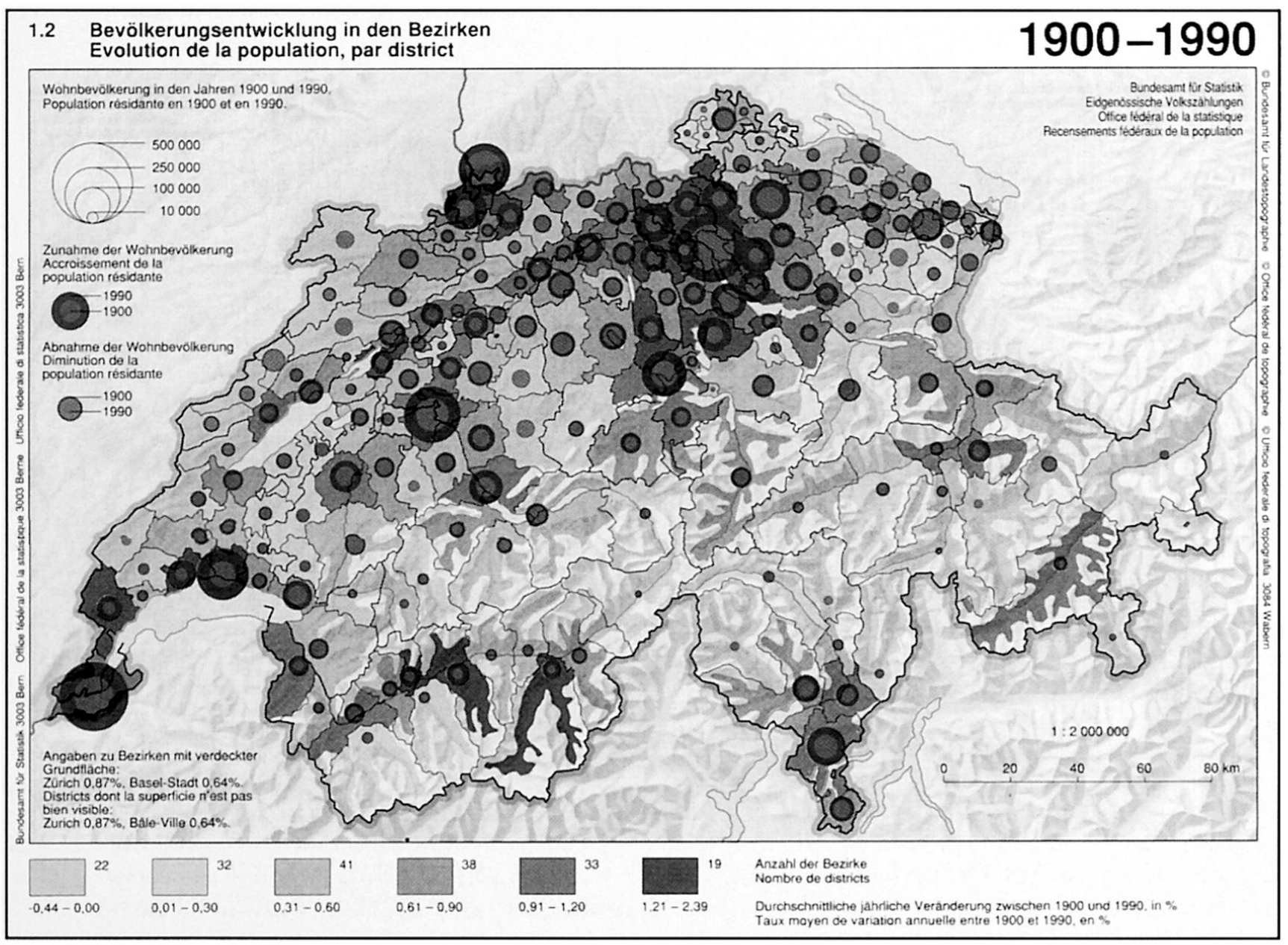

Abb. 2: Kartensynthese: Bevölkerungsentwicklung in den Bezirken, 1900-1990 
Sachverhalte unter Einschluß des Objektattributs Zeit zu gelangen. Bereits gegen Ende der siebziger Jahre wurden rechnergestützte Möglichkeiten erprobt, die Abbildung dynamischer Raumphänomene mit Mitteln der Computeranimation nicht nur zu erzeugen, sondern auch abzuspielen (MOELLERING 1980). ${ }^{3}$ Unter Einsatz einer dem seinerzeitigen Entwicklungsstand der Informationstechnik entsprechenden Großrechnerumgebung wurden dreidimensionale Zeitreihenkarten generiert, als Videosignale aufgezeichnet und anschließend in Echtzeit als Bewegtbild (1 Sekunde pro Zeitpunktkarte) auf einem Fernsehmonitor ausgegeben. ${ }^{4}$ Die Dynamik raumzeitlicher Sachverhalte wird durch explizite Einbeziehung der Zeitdimension in die Kartenmodellierung visualisiert, woraus - im Unterschied zur statischen Karte - eine sachgerechte Abbildung der Zeitattribute von Geodaten resultiert. Das Applikationspotential der Computeranimation in der thematischen Kartographie wurde von MOELLERING an mehreren Animationen dreidimensionaler Raum-Zeit-Karten erprobt, die Bevölkerungswachstum, Diffusionsprozesse oder Geländemodelle visualisieren. Diese Anwendungen können zugleich als frühe Beispiele multimedialer Karten gelten, da neben der Graphik auch die Datentypen Zeit und Ton zur multidimensionalen und multiperspektivischen Abbildung raumbezogener Daten eingesetzt werden. Nach MOELLERING kann die kartographische Computeranimation auch zur wissenschaftlichen Analyse raumzeitlicher Prozesse eingesetzt werden. Hierbei ist der Kartennutzer allerdings auf die dargebotenen und vom Operateur manipulierten Bewegtbilder beschränkt, eine unmittelbare Nutzerinteraktion mit der animierten Raum-Zeit-Karte findet nicht statt.

Im Zusammenhang mit dem rechnergestützten Konzept der Datenvisualisierung haben jüngere Studien den Einsatz von Animationstechniken in der thematischen Kartographie zur Visualisierung und Auswertung raumzeitlicher Sachverhalte thematisiert. Unter Einsatz der Computergraphik hat MONMONIER $(1989,1990,1992 a, b)$ das kartographische Potential der Visualisierung multivariater raumzeitlicher Daten mittels sogenannter Graphic scripts untersucht. Diese Methodik verwendet ebenfalls filmische Techniken zur Erzeugung von Bildsequenzen, die Karten, Fernerkundungsdaten, Diagramme oder Textinformationen enthalten können. Die Bildsequenzen lassen sich zu multiplen Zeitreihensichten zusammenfassen. Diese sogenannten Touren ermöglichen eine wesentlich sachgerechtere raumzeitliche Exploration der kompletten Kartendatenbank, als dies Einzelkarten oder Kartensynthesen vermögen. Graphic scripts bilden die methodische Basis für das «Atlas Touring»-Konzept MONMONIERS, einer graphikgestützten Präsentations- und Analyseumgebung raumzeitlicher Daten. ${ }^{5}$ Nach MONMONIER (1992a, b) vermag in erster Linie allerdings der fachlich geschulte Kartennutzer die gesamte Auswerte- und Analysemöglichkeiten für Raum-Zeit-Untersuchungen auszuschöpfen. In Weiterführung der Studien MONMONIERS untersuchen MacEACHREN/DiBIASE (1991) das Potential rechnerge- stützter Animation als graphikorientiertes Verfahren, das dem Kartographen nicht nur eine adäquate Informationspräsentation ermöglicht, sondern auch eine graphikgestützte Exploration der raumzeitlichen Daten des Nutzers unterstützt.

\section{Elektronische Diffusion statistischer Daten}

Für den fachlich geschulten Nutzer wie für die breite Öffentlichkeit hat das BFS raumbezogene Massenstatistik bislang in statischen Zeitpunkt- und Bilanzkarten visualisiert und in den bekannten BFS-Publikationen, darunter dem Statistischen Jahrbuch, veröffentlicht. Diese gedruckten Papierkarten ermöglichen die Betrachtung, optische Auswertung und weitere Interpretation der abgebildeten Datensätze. Im Sinne der kartographischen Kommunikation stellt dieses Verfahren eine Simplexkommunikation zwischen Produkt und Nutzer dar. Die Produktion der Zeitpunktkarten basiert seit längerem auf dem Einsatz mikrocomputergestützter Desktop-Mapping-Programme und Kartenkonstruktionspakete mit digitaler Dateneingabe, interaktiver Datenverarbeitung und analoger Graphikausgabe. Zur Verbesserung einer publikumsorientierten Diffusion geostatistischer Daten prüft das BFS, inwieweit das bisherige Informationsangebot durch interaktive multimediale Komponenten zu ergänzen ist. In diesem $\mathrm{Zu}$ sammenhang wurde ein interaktives Offline-Karteninformationssystem auf Mikrocomputerbasis erarbeitet (SCHLÜNZEN 1995). ${ }^{6}$ Die Konzeption dieses «ThemaKart»-Prototyps wurde in einer Forschungs- und Entwicklungsstudie unter Beteiligung der Autoren entwickelt (HERRMANN/ASCHE 1995).

\subsection{Multimediale Systemplattform}

Wesentliche Zielsetzung der Auswahl einer multimedialen Systemplattform für die elektronische Diffusion raumzeitlicher Statistik war es, ein gleichermaßen leistungsfähiges und wirtschaftliches Komplettsystem aus Standardkomponenten kommerzieller Hard- und Software zu konfigurieren.Um einen operationellen Einsatz und eine Übertragbarkeit des Prototyps zu gewährleisten, blieben sowohl Low-cost- als auch High-end-Kom-

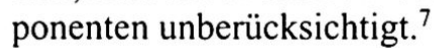

Kernbestandteile der ThemaKart-Konzeption wurden mit dem kommerziellen Autorenprogramm Macromedia Director, das als plattformübergreifender Quasistandard für multimediale Entwicklungsumgebungen gilt, und der Apple-Systemerweiterung Quicktime, die zur Erstellung der Kartenanimationen diente, in einen lauffähigen Prototyp umgesetzt, erprobt und evaluiert. Für den Import der digitalen Kartengrundlagen in die Animationsplattform Director war eine interaktive Nachbearbeitung aller Karten notwendig. Um einen raschen Bildaufbau sicherzustellen, wurde u. a. eine Farbreduktion vorgenommen, da der Karteninhalt mit jeder neuen Bildschirmkarte komplett wechselt und der Ausgangsdarstellung nicht hinzugefügt wird. 
Außerhalb der jeweils angezeigten Bildschirmkarte wurden interaktive Schaltflächen angeordnet, mit denen der Nutzer die Ablaufgeschwindigkeit sowie Beginn und Ende der Kartenanimation individuell festlegen kann; minimal ist der dynamische Vergleich zwischen zwei Dekaden möglich (Abb. 3). Um einzelne Zeitpunktkarten zu betrachten, läßt sich die Animation an jeder beliebigen Stelle anhalten. Zu jeder Standbildkarte können dann aus der Datenbank weitere Zusatzinformationen aktiviert werden. Um eine funktionsfähige Umgehung der Director-Programmbegrenzungen zu erhalten, wurde das Gesamtsystem in mehrere Module zerlegt. Weil bei der Nutzung das komplexe Gesamtsystem nicht im Hintergrund gehalten werden muß, ist die Handhabung einer großen Anzahl aktiver Bildelemente bei einer noch vertretbaren Länge der Systemreaktionszeiten möglich. Bei Wechsel in andere Programmodule ist ein rasches Nachladen gewährleistet. Auf diese Weise wird dem Benutzer der Eindruck vermittelt, mit dem System in near real time zu kommunizieren.

\subsection{Informationsvermittlung durch Kartenanimation}

Um die raumzeitliche Dynamik statistischer Massendaten unter Nutzung multimedialer Systemtechnik kartographisch adäquat wiederzugeben, enthält der ThemaKart-Prototyp ein interaktives Animationsmodul mit insgesamt acht unterschiedlich modellierten Kartenanimationen positions- und flächenbezogener Informatio- nen (Abb. 4). Durch Einbettung der temporal animierten Karten in das Rahmenkonzept eines multimedialen Statistikinformationssystems wird der Nutzer in die Lage versetzt, Thema, Umfang und Dauer sowie Start- und Zielpunkt der benutzten Geostatistik individuell zu bestimmen. Dies ermöglicht erstmals eine Duplexkommunikation zwischen Nutzer, Karte und Daten und dadurch weitreichende Auswerte- und Interpretationsmöglichkeiten der statistischen Daten.

\section{Animierte Zeitreihenkarten der Bevölkerungsstatistik}

Exemplarisch wurde das Visualisierungs- und Kommunikationspotential animierter Karten unter Nutzung ausgewählter Datensätze zur Bevölkerungsentwicklung der Schweiz von 1850 bis 1990 auf Kantons-, Bezirks- und Gemeindeebene erprobt. Vorbereitend wurden die $\mathrm{Da}-$ tensätze, deren zeitliche Tiefe fast ausnahmslos den Volkszählungsdekaden entspricht, mit dem Kartenkonstruktionspaket cart/o/graphix als separate Zeitpunktkarten modelliert. Dabei orientiert sich die Datenvisualisierung an den Modellierungslösungen der klassischen BFS-Karten für das Statistische Jahrbuch der Schweiz (SJB). Wie bei statischen Themakarten steht auch bei animierten Themakarten die effiziente Vermittlung raumbezogener Informationen im Vordergrund. Daher folgt die Datenvisualisierung den bekannten themen-
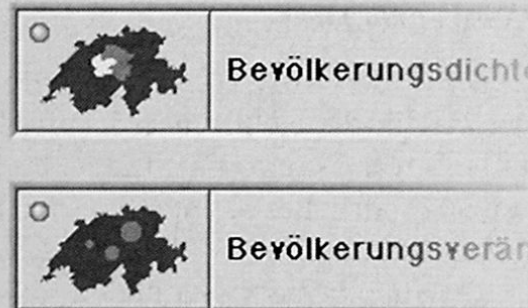

Beyölkerungsłerä!

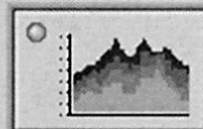

Beyölkerungsyerär

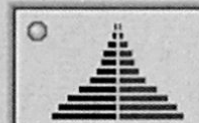

Bevölkerungsyerteil

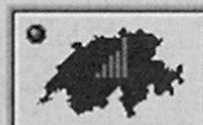

Entwicklung des B
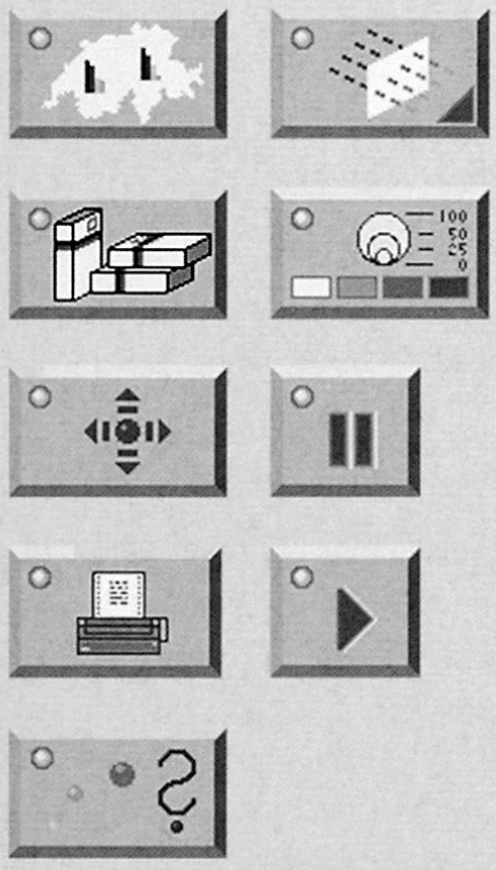
kartographischen Grundsätzen, sie hat zugleich aber auch Grundsätze der multimedialen, interaktiven Datenpräsentation zu beachten (ASCHE 1995). ${ }^{8}$ Aufgrund der dynamischen Informationspräsentation und der daraus resultierenden kurzen Betrachtungsdauer ist hierbei der Kartenwahrnehmung besondere Aufmerksamkeit zu widmen (OPENSHAW/WAUGH/CROSS 1994). Sie kann durch eine optische Akzentuierung der Datenveränderung verbessert werden.

\subsection{Visualisierung animierter Kartenobjekte}

Anders als bei filmischen Animationen, bei denen eine umfassende Gestaltung aller Animationskomponenten (Bildausschnitt, Bildperspektive, Beleuchtung, Ton u.a.) üblich ist, beschränkt sich die ThemaKart-Animation auf die graphische Gestaltung der Kartenobjekte als der wesentlichen Informationsträger. Zur Ermittlung wirksamer Modellierungslösungen für eine sichere Wahrnehmung der Karteninformationen wurden alternative
Visualisierungen positionsbezogener Wertverteilungen, Wertveränderungen und Wertentwicklungen erstellt. Anhand der Wahrnehmbarkeit der dargebotenen raumzeitlichen Informationen wurde deren Kommunikationspotential in informellen Tests mit BFS-Mitarbeitern und potentiellen Systemnutzern evaluiert. Prüfkriterium war die Möglichkeit, die Wertveränderungen der Bevölkerungsstatistik in ihrem Raummuster wahrnehmen und zeitlich zuordnen zu können.

Graphische Anpassungen an die dynamische Kartenpräsentation betrafen $\mathrm{u}$. a.:

a) positionsbezogene Wertverteilungen

Die Modellierung quantifizierter Positionsinformationen wurde in der Animation Bevölkerungsverteilung nach Gemeinden untersucht. Zunächst wurden für jeden Zeitpunkt die absoluten Zahlenwerte der über $3000 \mathrm{Ge}-$ meinden durch wertproportionale gleichfarbige Mengensignaturen symbolisiert. Die Aggregation aller Zeitpunktkarten zur Animation zeigt, daß wertproportionale

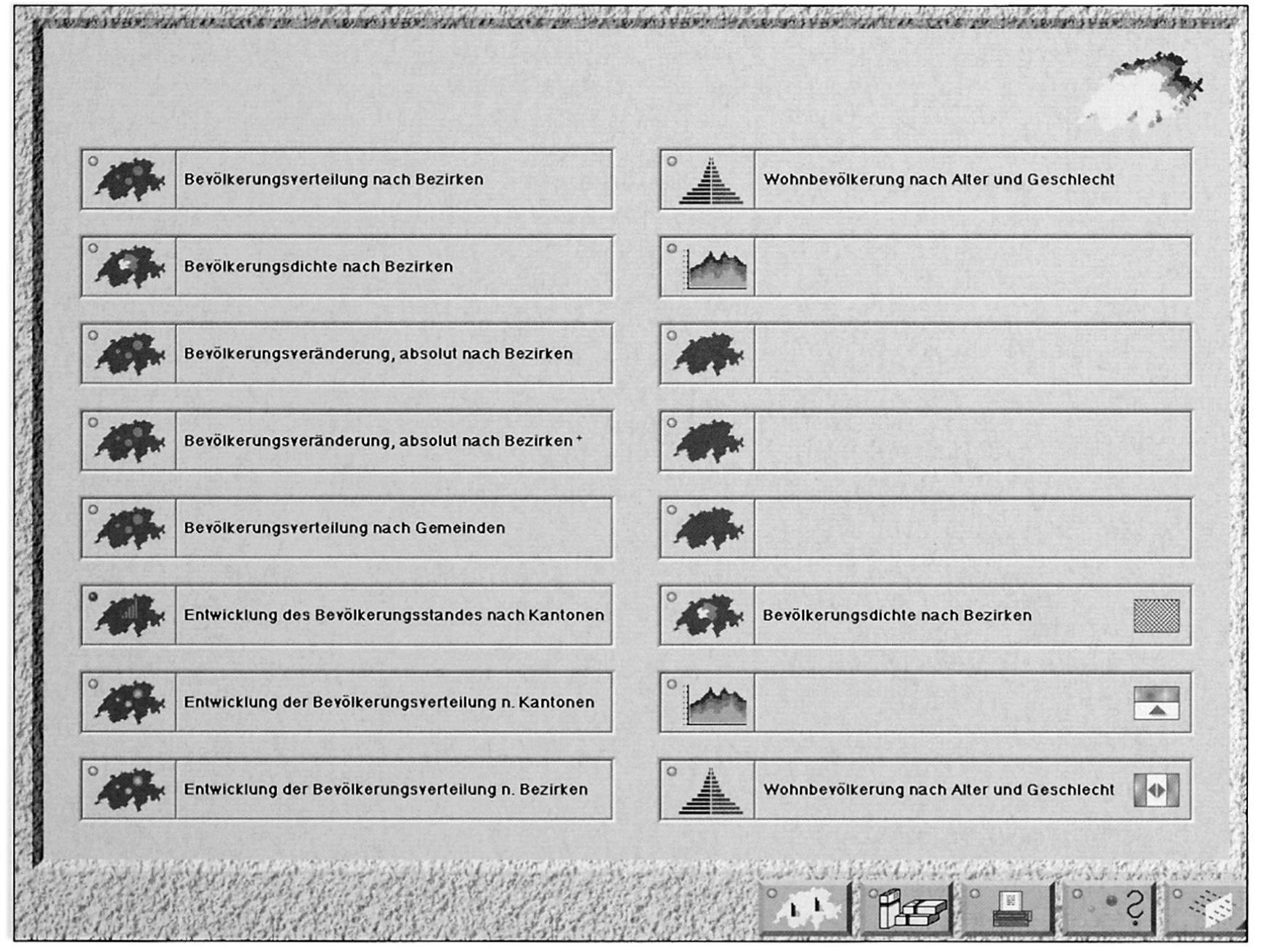

Abb. 4: Auswahldisplay der THEMAKART-Animation 

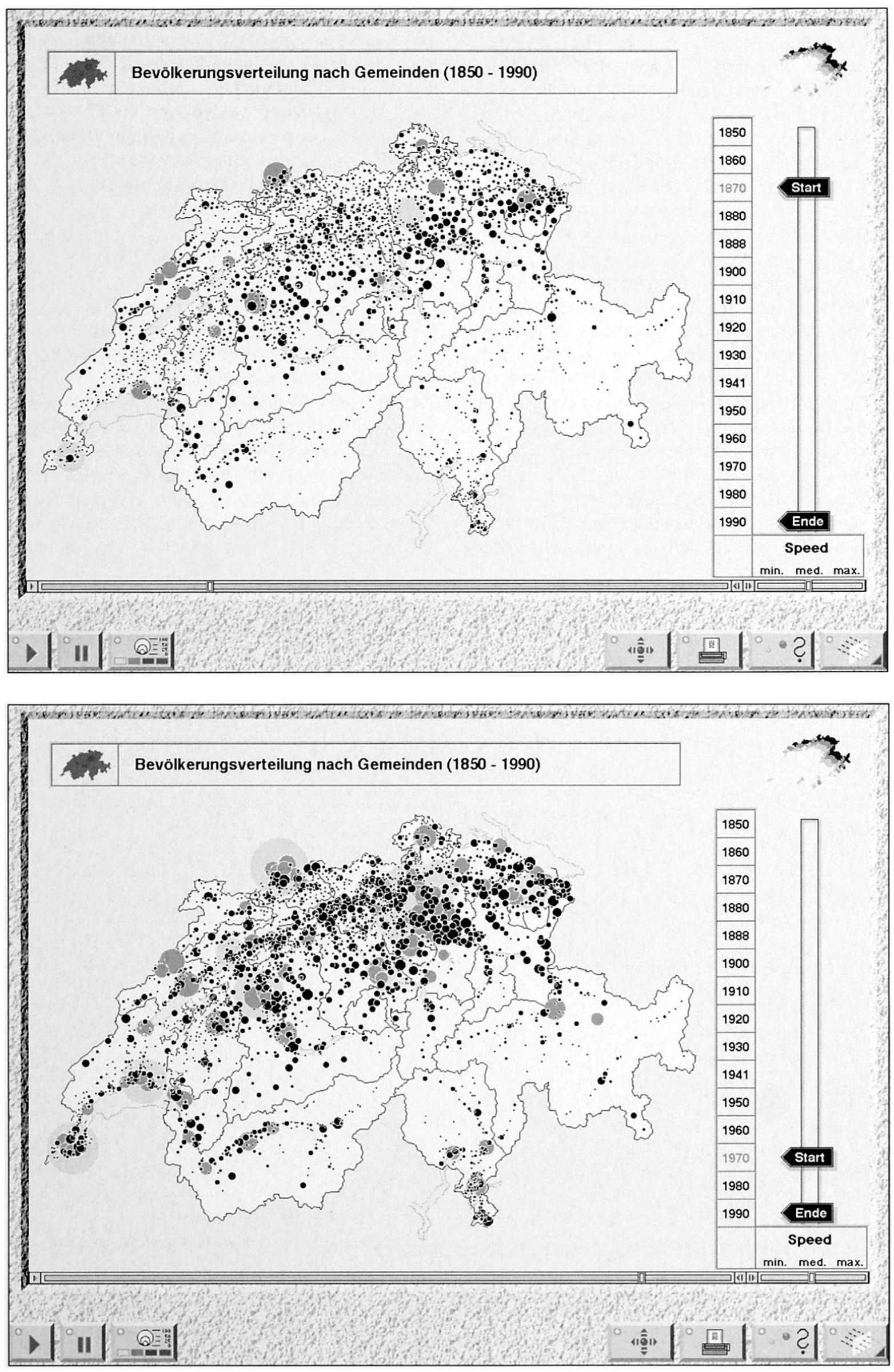

Abb. 5: Bevölkerungsverteilung nach Gemeinden; oben: 1870; unten: 1970 
Signaturenveränderungen bei einer derart großen Zahl positionsbezogener Einzelzeichen, die sich zudem häufig überlagern, aufgrund der kurzen Betrachtungszeit nur ungenügend wahrgenommen werden können. Um sowohl die Wahrnehmbarkeit der quantitativen Informationen zu verbessern als auch den notwendigen raschen Datenüberblick zu gewährleisten, wurden die Einzelwerte alternativ in drei Wertstufen gegliedert. Die so erhaltene Wertzusammenfassung wurde durch eine gegenläufige Intensitätsskala (kleiner Wert - intensive Farbe) symbolisiert. Auf diese Weise treten die Wertmaxima optisch zurück und können von kleineren, intensiv getönten Signaturen überlagert werden, ohne daß der jeweilige Positionsbezug aufgegeben wird. Während des Animationsablaufs kann der Nutzer sowohl jeden Einzelwert als auch dessen Stufenzugehörigkeit ablesen und so raumzeitliche Wertveränderungen erfassen. Damit ist sowohl die Detailanalyse von Einzelwerten als auch die Ableitung regionaler Wertverteilungen gewährleistet (Abb. 5).

\section{b) arealbezogene Wertveränderungen}

Die adäquate Wiedergabe flächenbezogener Wertveränderungen war Gegenstand der Animation Entwicklung
Bevölkerungsverteilung nach Bezirken. Hierzu wurden die Ausgangswerte in Kartodiagrammform als farbneutrale Proportionalkreise visualisiert, deren Größendimensionen denen des Bevölkerungsstandes entsprechen. Im Bewegtbild können Bevölkerungszu- und -abnahmen beliebiger Dekaden durch verschiedenfarbige Wachstums- und Schrumpfungskreise wiedergegeben werden, die in der Animationssequenz dem Bezugskreis (Wert 1850) jeweils hinterlegt bzw. überlagert werden. Sowohl das Oszillieren der Signaturengröße wie der Farbwechsel zwischen $\mathrm{Zu}$ - und Abnahme während des Animationsablaufs ermöglicht eine wirksamere Vermittlung der raumzeitlichen Entwicklung der flächenbezogenen Bevölkerungsverteilung als eine statische Kartenfolge der $\mathrm{Zu}$ - und Abnahmen oder Salden.

c) arealbezogene Wertvergleiche

Die effiziente Visualisierung von Wertbilanzen wurde in der Animation Entwicklung des Bevölkerungsstandes nach Kantonen erprobt. Zunächst wurde die Zu- und Abnahme der absoluten Bevölkerungsveränderungen in flächenbezogenen Stabdiagrammen für jeden Zeitpunkt visualisiert. Die Verknüpfung der Zeitpunktkarten zur Bildsequenz ermöglicht zwar die Wahrnehmung der

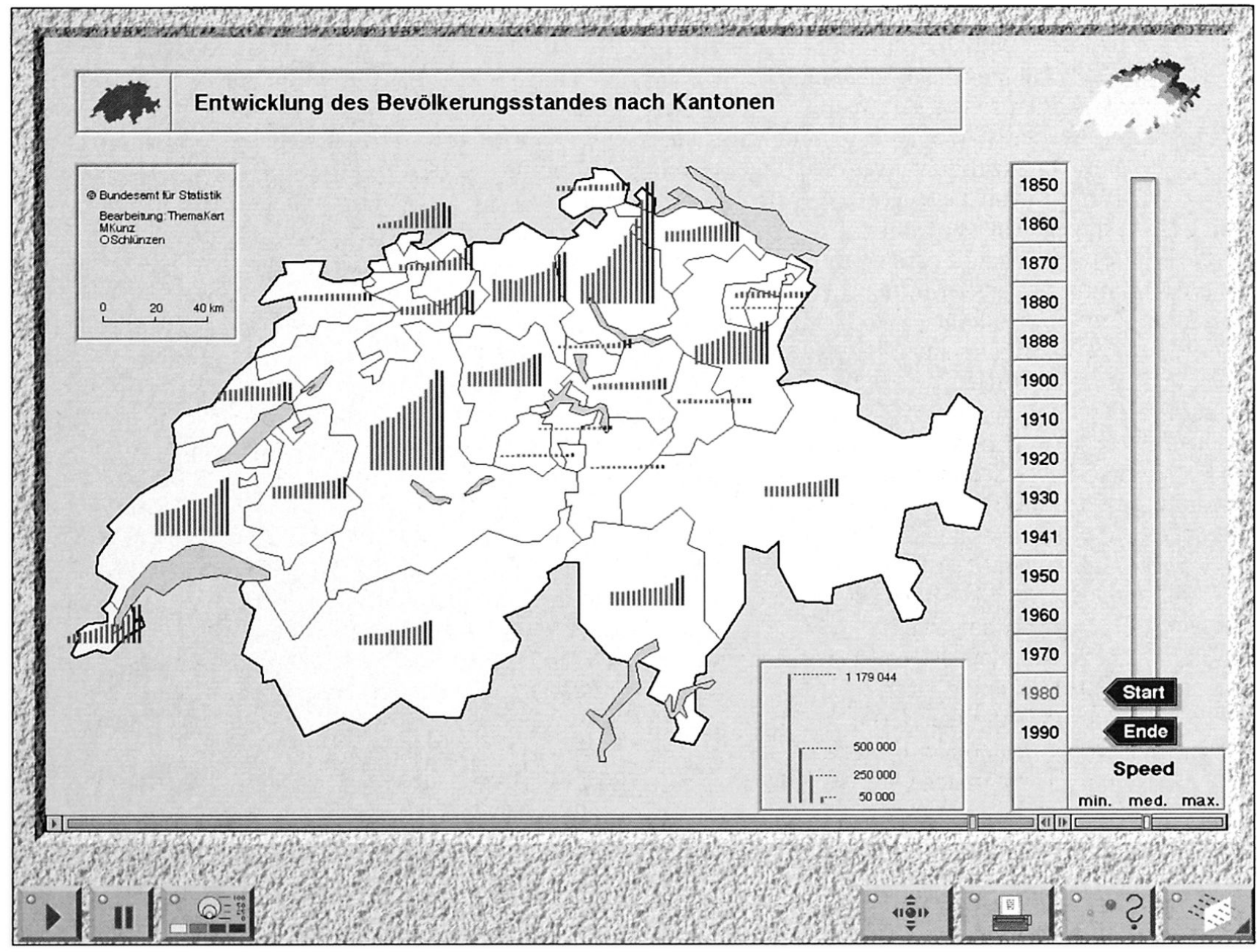

Abb. 6: Dynamisches Kartogramm, Stabdiagramm: Entwicklung des Bevölkerungsstandes nach Kantonen (junge Werte intensiver) 
zeitlichen Wertveränderung, nicht aber den Vergleich mit dem Ausgangswert. Indem im Animationsablauf der Ausgangsstab (Wert 1850) erhalten bleibt und die nachfolgenden Wertveränderungen durch Hinzufügung von Stäben veranschaulicht werden, kann der Nutzer sowohl die Wertentwicklung erkennen als auch Wertvergleiche anstellen (Abb. 6). Zur optischen Betonung der jüngeren Werte gegenüber dem Bezugswert sind die entsprechenden Stäbe intensiver eingefärbt.

\subsection{Modellierung des Animationsprozesses}

In animierten Karten bieten sich bei konstant gehaltener Animationsdauer grundsätzlich zwei Wege der Informationsvermittlung. Zum einen kann der Fokus auf einer kontinuierlich-dynamischen Informationspräsentation des Prozeßablaufs liegen. Hierzu ist es erforderlich, mittels Keyframe-Animationstechnik zwischen den vorhandenen Volkszählungsdekaden (Key frames) weitere Zeitpunktkarten linear, z. B. in Jahresschritten, zu interpolieren und in die Animationssequenz einzubinden. Das Ergebnis ist eine stufenlos ablaufende temporale Kartenanimation, bei der die raumzeitlichen Veränderungen kontinuierlich visualisiert werden. Zum anderen kann die raumzeitliche Veränderung im Prozeßablauf betont werden. Durch die Anwendung der Frame-byframe-Technik werden lediglich die Zeitpunktkarten der vorhandenen Volkszählungsdekaden ohne Interpolation von Zwischenschritten zu einer Animation miteinander verknüpft. Das Ergebnis ist eine temporale Kartenanimation, in der die raumzeitlichen Veränderungen durch optische «Sprünge» von Datensatz zu Datensatz sequentiell wiedergegeben werden (Abb. 7).

Um die Wirksamkeit beider Animationsverfahren für die dynamische Informationsvermittlung zu untersuchen, wurden im Zuge der Erstellung des ThemaKart-Prototyps alternative Visualisierungen des Datensatzes $\mathrm{Be}$ völkerungsdichte nach Bezirken erstellt. Zunächst wurde der flächenbezogene Datensatz als dynamisches Flächenkartogramm (Choroplethenkarte) visualisiert. Hierzu wurden die geostatistischen Ausgangsdaten in neun Wertstufen klassifiziert und in der Karte durch eine gelbviolette Intensitätsskala wiedergegeben (Abb. 8). Um trotz des administrativen Datenbezugs die räumliche Verbreitung der Bevölkerung insbesondere im Oberland zutreffend abzubilden, wurde bei z.T. extremen Differenzen zwischen Bezugs- und Verbreitungsfläche innerhalb der entsprechenden Bezirke nur die besiedelte Fläche der Talungen farbkodiert (Abb. 9). Anschließend wurden alternative temporale Kartenanimationen jeweils nach dem Frame-by-frame- und Keyframe-Verfahren erstellt.

Bereits ein einfacher visueller Vergleich beider Kartenanimationen durch Parallelablauf zeigt, da $\beta$ die Wahrnehmung der dynamischen Entwicklung der Bevölkerungsdichte durch den sequentiellen Ablauf der Frame-by-frame-Animation signifikant erleichtert wird, da jedes Einzelbild bei gleicher Ablaufdauer länger betrachtet werden kann (Wechselratendauer) als bei der kontinuierlichen Animation. Informelle Tests mit BFSMitarbeitern und potentiellen Systemnutzern belegten diese Einschätzung. Kontinuierliche Bildveränderungen durch Interpolation von Zwischenschritten und Überblendungen, wie sie $z$. B. bei Fotoanimationen eingesetzt werden, sind für die Animation statistischer Zeitreihenkarten wenig geeignet, da sie eine effiziente Vermittlung des Karteninhalts nicht unterstützen. Aus diesem Grund wurde allen Kartenanimationen die Frame-by-frameTechnik zugrunde gelegt.

\section{Nutzungspotential animierter Statistikkarten}

Der Einsatz kartographischer Animationstechniken verbessert nicht nur die fachgerechte Visualisierung raumzeitlicher Massendaten in thematischen Karten. Durch die Anwendung interaktiver Informationstechniken wird die Kartenpräsentation zugleich stärker am multimedial beeinflußten Wahrnehmungsverhalten der Kartennutzer ausgerichtet. Verglichen mit konventionellen statischen Themakarten wie jenen des SJB, erweitern multimedial animierte Kartengraphiken folglich nicht nur das Modellierungs-, sondern auch das Nutzungspotential thematischer Karten. Dies bedeutet im einzelnen: a) Die Möglichkeit der Interaktion mit dem System eröffnet dem Nutzer eine individuelle, auf die einzelne Anwendung orientierte Informationsentnahme. Damit kann der Nutzer Art, Umfang, Abfolge, Raumbezug und Geschwindigkeit der ausgewählten Statistik- und Kartendaten bestimmen und zwischen ihnen navigieren. Der ThemaKart-Prototyp ermöglicht unterschiedliche interaktive Informationsnutzungen.

b) Trotz wesentlich erweiterten Datendargebots erhält der Nutzer - leichter und zugleich umfassender als bei vergleichbaren Printprodukten wie z. B. dem SJB - im ThemaKart-Prototyp einen Überblick über die verfügbaren Statistikdaten sowie deren Raum- und Zeitbezug. Dies versetzt ihn in die Lage, rascher, gezielter und individueller auf die ihn interessierenden Daten zugreifen und diese vorwiegend visuell auswerten zu können.

c) Die enthaltenen Kartenanimationen versetzen den Nutzer in die Lage, Statistikzeitreihen als dynamische raumzeitliche Prozesse erfassen und in Ablauf und Entwicklung analysieren zu können. Trotz Informationsverdichtung gegenüber konventionellen Kartenfolgen, wie sie z. B. das SJB enthält, trägt die der Prozeßhaftigkeit der Statistikdaten angepaßte kartographische Visualisierung zu verbesserter Auffaßbarkeit und Auswertbarkeit der Statistikdaten bei.

d) Der Nutzer kann unterschiedliche Raumbezüge der Statistikdaten (Gesamtschweiz, Kanton, Bezirk und Gemeinde) auswählen - anders als im SJB, welches diese Informationen nur in Auswahl und ohne direkten Raumbezug enthält. Ferner kann er den Zeitbezug der dargebotenen Statistikdaten verändern, um spezifische Entwicklungsphasen innerhalb des Gesamtprozesses besser verfolgen und studieren zu können. Bei der gemeindebezogenen Bevölkerungsstatistik kann so z. B. 


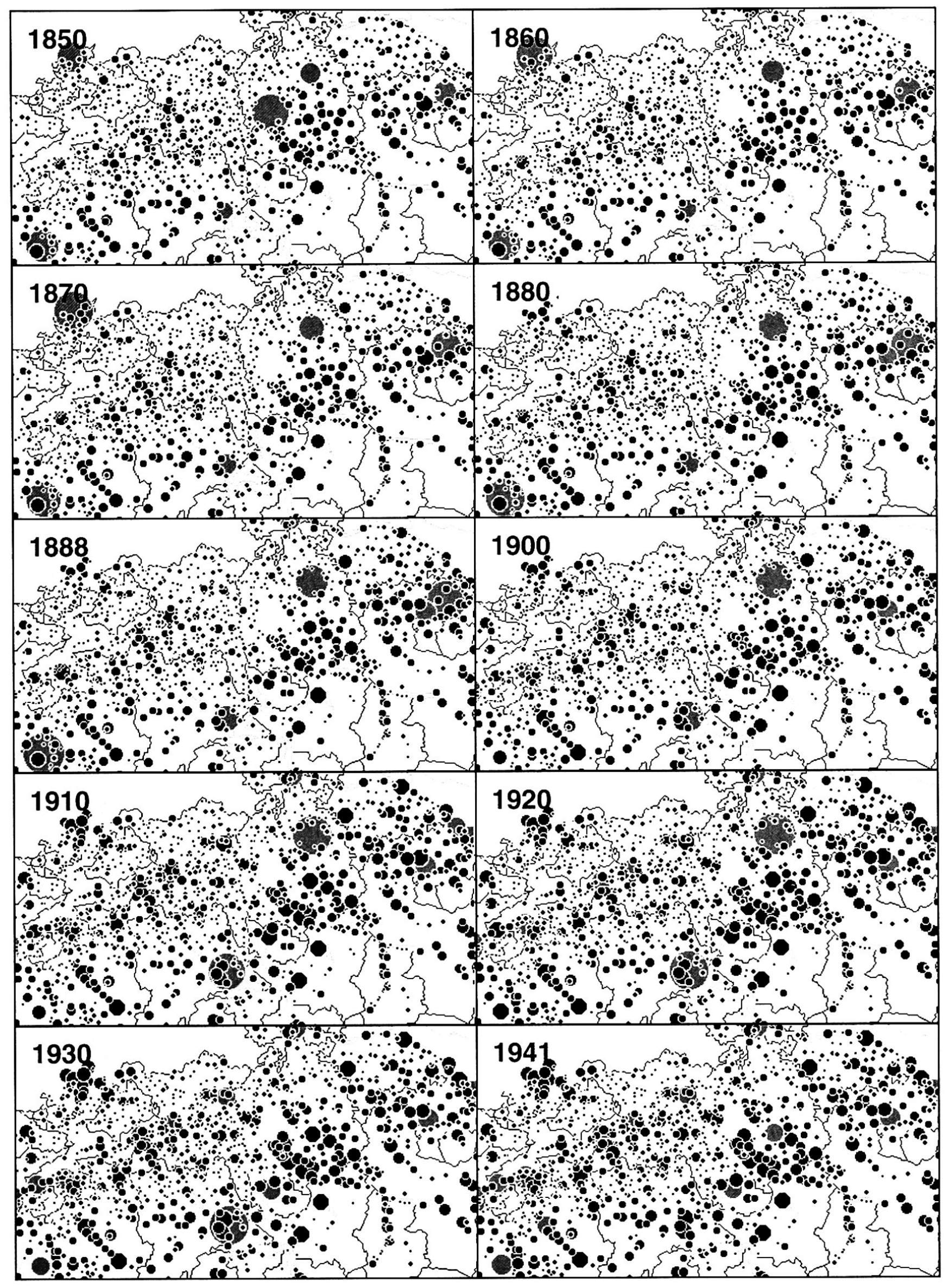

Abb. 7.1: Bevölkerungsverteilung nach Gemeinden (1850-1941) 


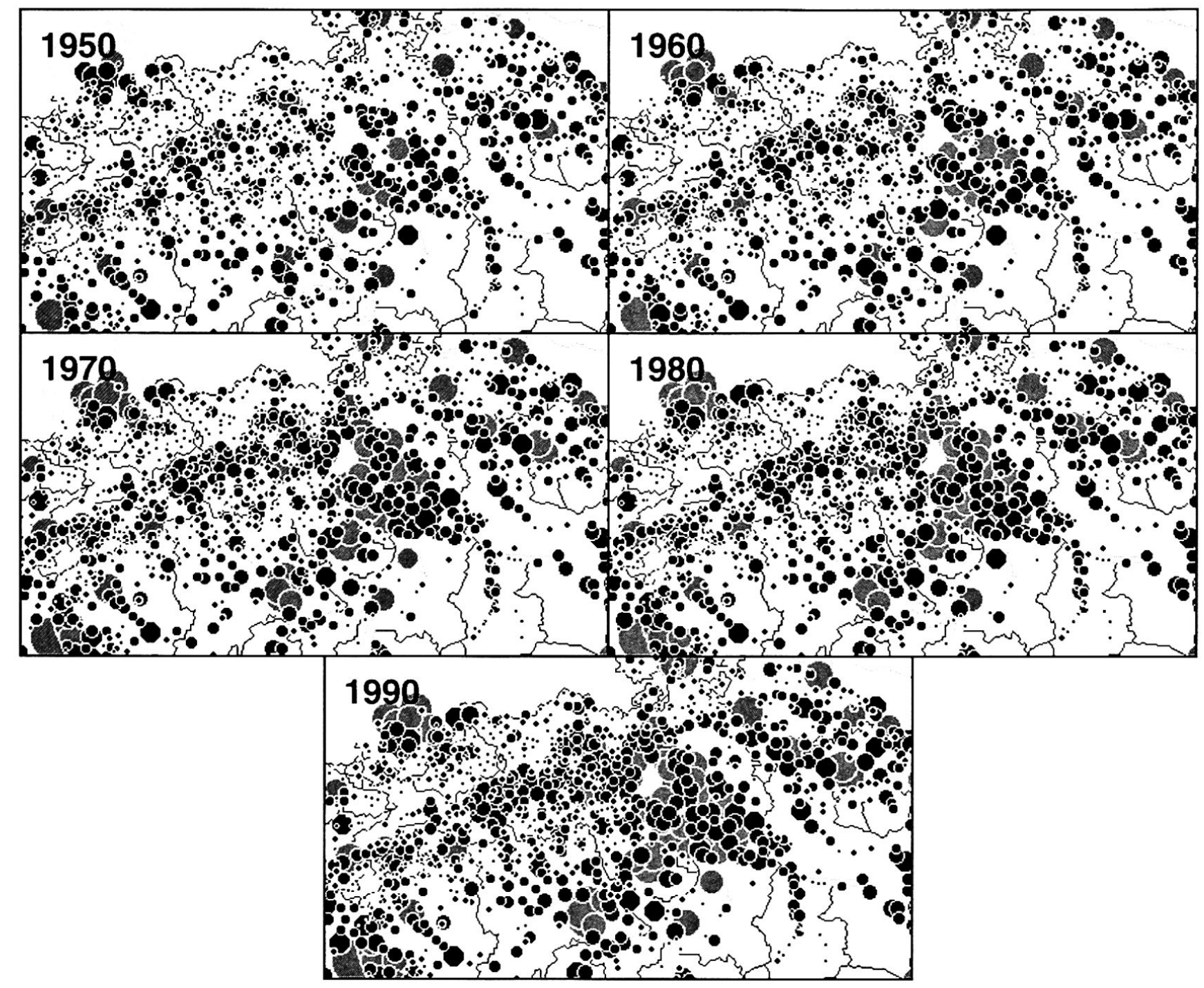

Abb. 7.2: Bevölkerungsverteilung nach Gemeinden (1950-1990)

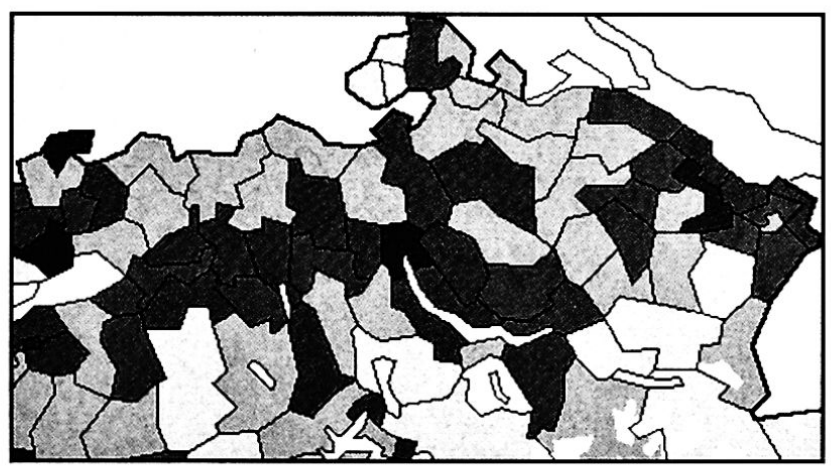

Abb. 8: Bevölkerungsdichte nach Bezirken 1960 (Dichtewerte bedecken die gesamte Gemeindefläche)

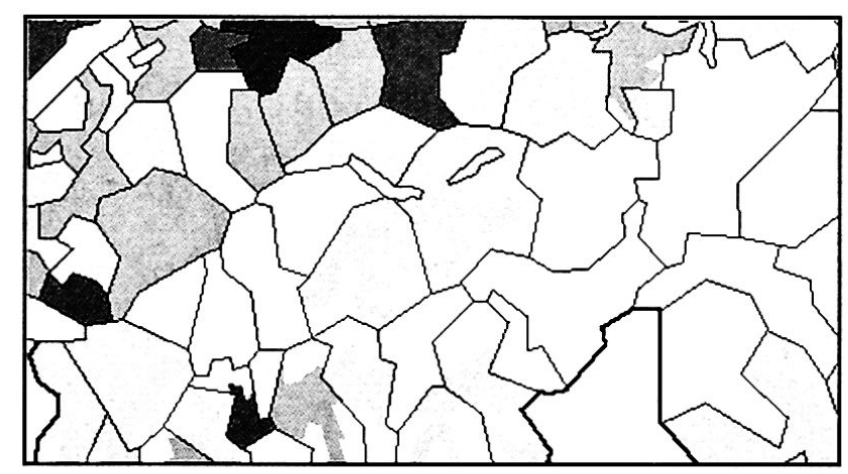

Abb. 9: Bevölkerungsdichte nach Bezirken 1960 (Dichtewerte bedecken die gesamte Gemeindefläche) 
der Bau der Gotthardbahn 1870-80 oder der Rückgang der Stadtzürcher Bevölkerung 1970-80 unmittelbar anschaulich gemacht werden. Statische Printprodukte wie die Karten des SJB bieten keine vergleichbaren Betrachtungsmöglichkeiten und Auswertehilfen.

\section{Schlußbemerkungen}

Zweifellos erfordert eine operationelle Anwendung der in der Kartographie bislang wenig genutzten Visualisierung statistischer Massendaten in Form animierter Themakarten weitere Forschungs- und Entwicklungsaktivitäten, nicht zuletzt im Bereich der Kartengraphik. Eine vorläufige Bewertung der im Rahmen des ThemaKartPrototyps erstellten Kartenanimationen raumzeitlicher Bevölkerungsstatistik erlaubt allerdings die Schlußfolgerung, daß animierte thematische Karten dem Nutzer umfangreiche interaktive Informations- und Auswertemöglichkeiten raumzeitlicher Zusammenhänge bereitstellen. Aufgrund der dynamischen Visualisierung raumzeitlicher Massenstatistik und der interaktiven Datenexploration übertreffen sie das Auswertepotential statischer Printkarten signifikant. Dabei erweitert kartographische Animation das Spektrum fachgerechter Datenvisualisierung und fördert so das vertiefte Verständnis raumzeitlicher Entwicklungen. Teilweise erst durch die kartographische Animation bevölkerungsstatistischer Daten erschließen sich dem Nutzer ungekannte inhaltliche und räumliche Überblicke und Zusammenhänge, die herkömmliche Kartensynthesen oder Kartenfolgen aufgrund der statischen, auf die Einzelkarte optimierten Datenpräsentation nicht unterstützen. Die vorgestellte multimediale Aufbereitung statistischer Daten, ihre individuelle Nutzbarkeit und ihre elektronische Diffusion (offline-online) ermöglicht nicht nur erweiterte Karten- und Datennutzungen, durch sie wird auch ein erweiterter Nutzerkreis statistischer Daten erreicht. Damit vermag der ThemaKart-Prototyp schließlich einen Beitrag zur Transparenz und Akzeptanz amtlicher Statistik sowie der erforderlichen Datenerhebung, Datenverarbeitung und Datenverwaltung zu leisten.

\section{Danksagung}

Die Verfasser danken Herrn Dr. Hans Steffen (BFS, Bern), der seit Jahren mit Engagement und Umsicht die adäquate Visualisierung statistischer Massendaten gefördert hat. Mit seiner Unterstützung konnte im Winter 1994/95 das interaktive Karteninformationssystem ThemaKart des BFS als Offline-Prototyp entwickelt werden. Wesentlichen Anteil an den Entwicklungsarbeiten hat Dipl.-Ing. Olaf Schlünzen (Karlsruhe), der den Prototyp im Rahmen einer Diplomarbeit am BFS realisierte. Auch ihm sei für seine engagierte Mitarbeit gedankt. Dankend erwähnt wird ferner die Unterstützung, welche die Autoren auch von hier nicht namentlich erwähnten BFS-Mitarbeitern erhalten haben.

\section{Anmerkungen}

${ }^{1}$ Expertenvertrag ThemaKart 123.01.208.1 vom 25. 8.1994. Im Rahmen dieses Projektes wurde untersucht, wie das Potential der rechnergestützten interaktiven Kommunikation für die Speicherung, Vermittlung und vor allem Nutzung raumbezogener statistischer Massendaten eingesetzt werden kann.

2 Eine ausführliche Darstellung kartographisch relevanter Animationstechniken findet sich z. B. bei DRANSCH (1997). Im engeren Sinn bezeichnet der Begriff Animation die filmische Aufnahme und Wiedergabe einer Abfolge unbewegter Einzelgraphiken.

${ }^{3}$ Bereits 1978 hat MOELLERING hierzu ein Videoband vorgelegt, das verschiedene mit Musik unterlegte Animationen statistischer Oberflächen (u. a. Bevölkerungsentwicklung USA, Diffusion agrartechnischer Innovationen in Kalifornien) zeigt.

${ }^{4}$ Bei Echtzeitanimationen kann der konkrete Ablauf einer Bildsequenz durch den Operateur am Rechner direkt manipuliert werden. Die Manipulationsmöglichkeit rechnergestützter Animation betrachtet MOELLERING (1980) als wesentlichen Vorzug gegenüber der filmischen Animation, deren Bildsequenzen nach einem vordefinierten Ablauf lediglich abgespielt werden können.

5 Hierfür hat MONMONIER 1991 Beispiele ("US Daily Newspapers in the twentieth century", "Women in Politics" vorgelegt, die unterschiedliche dynamische Visualisierungen und Interaktionsmöglichkeiten demonstrieren.

6 In Ergänzung zur hier beschriebenen Offline-Version des ThemaKart-Informationssystems auf CD-ROM ist jüngst eine Online-Präsentation thematischer Karten des BFS realisiert worden (MANDERBACH 1997).

${ }^{7}$ Als Arbeitsplattform wurde hauptsächlich ein Apple Macintosh Ilvx, für Spezialaufgaben ein Apple Macintosh 8100 AV sowie Graphikperipherie genutzt, als Software wurden Macromedia Director (Autorenprogramm), cart/o/graphix $\| Q$ (Kartenkonstruktion), Aldus Freehand (Desktop Publishing), Adobe Photoshop (digitale Bildbearbeitung), Microsoft Excel 4.0 (Tabellenkalkulation), Adobe Premiere 3.0, Macromedia Soundedit (Spezialeffekte Animation) und weitere Programme eingesetzt.

${ }^{8}$ Aufgrund des experimentellen Charakters des ThemaKartPrototyps wurde die graphische Modellierung der Screenmaps im Vergleich zum Screendesign der Benutzeroberfläche prioritär behandelt.

\section{Literatur}

ASCHE, H., HERRMANN, C. M. (1993), Electronic mapping systems - a multimedia approach to spatial data use. In: Mesenburg, P. (Hrsg., 1993): Proc. 16th Intern. Cartogr. Conference, Köln, Bd. 2: 1101-1108. Bielefeld.

ASCHE, H., HERRMANN, C. M. (1994): Designing interactive maps for planning and education. In: MacEachren/Taylor 1994: 215-242.

ASCHE, H. (1996): Modellierung und Nutzung elektronischer Karten. In: Mayer, F., Kriz, K. (Hrsg.): Kartographie im multimedialen Umfeld. Wiener Schriften zur Geographie und Kartographie, Bd. 8: 150-167. Wien.

BERTIN, J. (1974): Graphische Semiologie. Berlin-New York. DiBIASE, D., MacEACHREN, A. M., KRYGIER, J. B., REEVES, C. (1992): Animation and the role of map design in scientific visualization. In: Cartography and Geographic Information Systems 19, 4: 201-214, 265-266. 
DORLING, D. (1992): Stretching space and splicing time: From cartographic animation to interactive visualization. In: Cartography and Geographic Information Systems 19, 4: 215-227.

DRANSCH, D. (1997): Computeranimation in der Kartographie. Theorie und Praxis. Heidelberg-Berlin.

HEARNSHAW, H.M., UNWIN, D.J. (Hrsg., 1994): Visualization in geographical information systems. Chichester. HERRMANN, C. M., ASCHE, H. (1995): Entwicklung und Bereitstellung einer digitalen interaktiven Kartenbibliothek auf der Basis animierter thematischer Karten aus dem Bereich der amtlichen Statistik. Expertenvertrag 123.01.208.1, unveröff. Abschlußbericht, Bern.

MCCORMICK, B.H., DeFANTI T.A., BROWN, M.D. (Hrsg., 1987): Visualization in scientific computing. Computer graphics 21, 6.

MacDOUGALL, E.B. (1992): Exploratory analysis, dynamic statistical visualization, and geographic information systems. In: Cartography and Geographic Information Systems 19, 4: 237-246.

MacEACHREN, A. (1994a): Time as a cartographic variable. In: Hearnshaw/Unwin 1994: 115-130.

MacEACHREN, A. M. (1994b): Visualization in modern cartography: setting the agenda. In: MacEachren/Taylor (1994): $1-12$.

MacEACHREN, A. M., DiBIASE, D.W. (1991): Animated maps of aggregate data. Conceptual and practical problems. In: Cartography and Geographic Information Systems, 18, 4: 221-229.

MacEACHREN, A.M., MONMONIER, M. (1992): Introduction. Geographic visualization. In: Cartography and Geographic Information Systems 19, 4: 197-200.

MacEACHREN, A.M., TAYLOR, D. R. F. (Hrsg., 1994): Visualization in Modern Cartography. Modern Cartography, 2. Oxford.

MANDERBACH, A. (1997): Online-Informationssystem auf der Basis interaktiver thematischer Karten aus dem Bereich der amtlichen Statistik (BFS Bern). Unveröff. Diplomarbeit Karlsruhe.

MOELLERING, H. (1980): The real-time animation of threedimensional maps. In: American Cartographer, 7: 67-75
MONMONIER, M. (1989): Graphic scripts for the sequenced visualization of geographic correlation. Proc. GIS/LIS '89, Orlando: 381-389.

MONMONIER, M. (1990): Strategies for the visualization of geographic time-series data. In: Cartographica 27, 1: 30-45. MONMONIER, M. (1992a): Summary graphics for integrated visualization in dynamic cartography. In: Cartography and Geographic Information Systems 19, 1: 237-246.

MONMONIER, M. (1992b): Authoring graphic scripts: experiences and principles. In: Cartography and Geographic Information Systems 19, 4: 247-260, 272.

OPENSHAW, S., WAUGH, D., CROSS, A. (1994): Some ideas about the use of map animation as a spatial analysis tool. In: Hearnshaw/Unwin 1994: 131-138.

PETERSON, M.P. (1995): Interactive animated cartography. Englewood Cliffs.

RASE, W.-D. (1974): Kartographische Darstellung dynamischer Vorgänge in computergenerierten Filmen. In: Kart. Nachr. 24: 201-215.

SCHLÜNZEN, O. (1995) Informationssystem ThemaKart. Entwicklung und Bereitstellung einer digitalen interaktiven Kartenbibliothek auf der Basis animierter thematischer Karten aus dem Bereich der amtlichen Statistik. Unveröff. Diplomarbeit Karlsruhe.

SIEBER, R., BÄR, H. R. (1996): Das Projekt «Interaktiver Multimedia-Atlas der Schweiz". In: Kartographie im Umbruch neue Herausforderungen, neue Technologien. Beiträge zum Kartographiekongreß Interlaken 96. Kartogr. Publikationsreihe Nr. 14, 211-226, o. O.

TAYLOR, D.R.F. (1991a) A conceptual basis for cartography / New directions for the information era. In: Cartographica 28, 4: 1-8

TAYLOR, D.R.F. (Hrsg., 1991b): The Microcomputer and Modern Cartography. Modern Cartography, 1. Oxford.

TAYLOR, D.R.F. (1994): Perspectives on visualization and modern cartography. In: MacEachren/Taylor 1994: 333-341. TOBLER, W.R. (1970): A computer movie simulating urban growth in the Detroit region. In: Economic Geography 46: 234-240.

WITT, W. (1970): Thematische Kartographie. Hannover. 


\section{Unheimliche Heimat $^{1}$}

\section{Einleitung}

Heimat ist die Identitätskategorie par excellence. Heimat kann für fast alles stehen, weil Assoziationen auf zeitlicher, kognitiver, struktureller, emotionaler und politischer Ebene hergestellt werden können. Die zugeordneten Kategorien lauten etwa Vergangenheit (Kindheit), Berge, Einfachheit, Kleinteiligkeit, Geborgenheit und Bauerntum. Der Historiker Kos fragt sich, ob «Heimat» für ihn nicht längst zu einer Konstellation geworden ist, "die sich aus sehr unterschiedlichen und disharmonischen Quellen speisen kann? Ein Modulsystem aus lauter Wahlverwandtschaften mit Orten, Filmen, Büchern, Menschen und Liedern? Das Leben, könnte man sagen, ist ein leerer Raum, den sich der Mensch mit Objekten der Zuneigung erst einrichten muß» (KOS 1993: 42). Die Tatsache, daß bestimmte Töne, Gerüche oder Farben mit an sich neutralen Eigenschaften einen Menschen «heimatlich» anrühren, daß sie freudige oder bedrückende Gefühle auszulösen vermögen, findet ihre Erklärung darin, daß sie irgendwann einmal im eigenen Leben mit einer freudigen oder bedrückenden Begebenheit verbunden gewesen sind. Das Ereignis selbst ist meistens längst vergessen. Es bleibt aber die erneute Auslösung dieser Gefühle durch die mit dem früheren Erlebnis verbundenen Gegenstände und Erscheinungen. So ist es möglich, daß die mit einem Musikstück verbundenen - vermeintlich bereits vergessenen - Erinnerungen, die wie von einer inneren Kamera als bewegte Bilder festgehalten wurden, durch das erneute Hören des Stücks wieder an die Oberfläche auftauchen und gleichsam wie ein private movie vor einem inneren Auge nochmals ablaufen.

Wie das funktioniert, hat THEwELEIT in einem Beitrag über Miles Davis beschrieben. Für die erneute Auslösung der Gefühle ist es nicht nötig, an den Ort zurückzukehren, an den diese Erinnerung gekoppelt ist. Es genügt, die Platte wieder zu hören. Für THEWELEIT spielt der Plattenspieler nicht nur ab, er nimmt auch auf. Schallplatten sind für ihn besondere Geschichtsspeicher. Auf Platten werden bestimmte Gefühle, die man beim Hören hatte, derart genau gespeichert, daß THEWELEIT dies nicht einfach nur Erinnerungen nennen will, auch nicht nur Hilfsmittel zur Wiederbelebung. «Die Platten haben etwas aufgezeichnet, während sie liefen; nicht nur etwas abgespielt. Zwischen Platte/Tonarm/Lautsprecher und aufnehmendem $\mathrm{Ohr} / \mathrm{Gefühlsstrom} \mathrm{scheint} \mathrm{sich}$ eine Aufnahmeapparatur gebildet zu haben zur Spei- cherung dieser Gefühlsströme (...). Will sagen, die Platten funktionieren als Speicher von Körper- und Wahrnehmungszuständen, als Indikatoren der Differenz, in der ich mich jetzt zu früheren Zuständen meines Lebens befinde» (THEWELEIT 1989: 82f.).

Heimat kann mit einer Art produktiver Gefühlserinnerung gleichgesetzt werden, die längst Vergessenes mittels diverser Speichermedien wie beispielsweise Schallplatten, Fotografien und Filmen über die verschiedenen Sinne wiederbelebt und erst dann rational bewältigt. Die Erinnerung hat unter anderem etwas mit physischer Kopräsenz an einem bestimmten Ort zu tun, wo sie lokalisiert ist. Damit gewisse Erinnerungen wieder aktiviert werden, kann man auch an den Ort ihres Ursprungs zurückgehen. Der Ort kann sich zwischenzeitlich völlig verändert haben, die Erinnerungen bleiben die gleichen. Man nimmt nicht nur den aktuellen Zustand eines Ortes wahr, sondern auch dessen Vergangenheit. Die Örtlichkeit wird im obengenannten Sinn zum Speichermedium, und Heimat umschreibt das faszinierende Gefühl der körperlichen Präsenz an einer ganz konkreten Örtlichkeit. Die einstige Wirklichkeit erhält sich aber nicht ebenso unverändert wie das ideelle, zudem meist beschönigte Abbild im Gedächtnis. Auch das gedanklich Bewahrte wandelt sich im Laufe der Zeit. Die Suche nach der verlorenen Zeit gilt demnach einer Wirklichkeit, die es womöglich nie so gegeben hat: Jeder Heimat haftet etwas Illusionäres an.

Die physische Präsenz an einem bestimmten Ort für ein entsprechendes Heimatgefühl ist das Bindeglied zwischen Geographie und Heimat, was auch mit dem populären Schlagwort «Globalisierung» kaum wegzudiskutieren ist. Erinnerung hat also etwas mit dem Fasziniertsein von der Zeitlichkeit der physischen Präsenz zu tun, und Heimat kann in diesem Sinne als etwas gesehen werden, was einem zu diesem Fasziniertsein verhilft. Womit auch für das konservierende Element von Heimat eine mögliche Erklärung gegeben werden kann: Man will eine Örtlichkeit, mit der man ganz bestimmte Erinnerungen verbindet, erhalten, damit diese Erinnerungen jederzeit wieder abrufbar sind, wenn man sich nur wieder an diesen Ort begibt.

Andreas Huber, Dr., Geographisches Institut, Universität Zürich Irchel, Winterthurerstraße 190, 8057 Zürich 
Der Heimatbegriff weist zweifellos in den Köpfen der meisten Menschen primär eine räumliche Komponente auf. Daran ist grundsätzlich nichts zu kritisieren. Problematisch aber wird es, wenn die Raumabstraktion Heimat dazu verwendet wird, regionalistische oder nationalistische Ziele zu legitimieren bzw. solche Erscheinungen zu erklären. Das Unheimliche an der Heimat liegt demnach darin, daß man als ihr wesentliches Merkmal eine psychische Bezogenheit zu übergeordneten Bezugssystemen attestiert: Bezogenheit zu einem Raum, zu einer Nation, zu einer Landschaft, zu einem Dorf oder einer Stadt, zu einem Volk, zu einer Gruppe, zu weiß ich was alles. Mit der Verknüpfung zwischen dem einzelnen, seiner individuellen Heimat - verstanden als persönliches Bezugssystem - und übergeordneten räumlichen oder ethnischen Bezugssystemen erhält der Heimatbegriff erst seinen mystischen Charakter. Heimat soll ein Geheimnis, ein Mysterium sein, weil eine übermäßige Bewußtmachung unter Umständen «urtümliche Kräfte» zerstören könnte, die leicht zu mobilisieren und zu steuern sind und die sich ausgezeichnet für politische Manipulationen eignen. Die Liebe zur Heimat muß diffus und mystisch bleiben, weil damit leichter von der konkreten sozialen Wirklichkeit abgelenkt werden kann. Soll der Heimatbegriff deshalb in seiner Unheimlichkeit belassen werden, weil damit besser blinde, nationalistische Emotionen entfacht werden können? ${ }^{2}$

\section{Heimat: ein vielfach verwendbarer Begriff}

Heimat als Gefühls- und Gemütswert, als Hort der Geborgenheit, der Vertrautheit und des Vertrauens ist in erster Linie eine unpolitische Größe. Faßt man Heimat als Mythos auf, dann wäre sie gemäß BARTHES, der Mythos als eine viel stärker durch ihre Absichten als durch ihren Buchstaben bestimmte Aussage definiert was für Heimat sicher zutrifft -, eine «entpolitisierte Aussage» (BARTHES 1964). Gerade das aber macht sie in höchstem Maße politisch manipulierbar. Die politische Manipulierbarkeit resultiert aus dem programmatischen Festhalten am bewährten Alten, dem Rückzug aus der Welt der Politik, wobei diese unpolitische Grundhaltung natürlich ebenfalls - zwar meist unreflektiert - eine politische Entscheidung ist. Heimat bietet daher in bezug auf politische Überzeugungen ein völliges Vakuum. Sie ist sozusagen allen Parolen kritiklos ausgeliefert, wie dies VORLÄNDER exemplarisch an der Heimatideologie des Dritten Reiches darstellt (VORLÄNDER 1984).

In der Schweiz ist die Diskussion um Heimat anläßlich des Kulturboykotts gegen die Jubiläumsfeierlichkeiten zum 700jährigen Bestehen der Eidgenossenschaft so richtig in Gang gekommen. Die Ereignisse im Umfeld dieser Feierlichkeiten (Fichenaffäre, Verlust an nationalem Konsens und politischer Kultur, EWR-Diskussion) ließen die Frage nach der Einstellung der Schweizerinnen und Schweizer zu ihrem Land und die Frage nach ihren Vorstellungen von Heimat aufwerfen. Zumindest wurde dies in den Medien so vermittelt. Exemplarisch dokumentiert ist diese Diskussion beispielsweise im Buch «Störfall Heimat - Störfall Schweiz», einer Publikation, die die Referate und Diskussionsbeiträge des 3. IAP-Forums zusammenfaßt. Darin werden von diversen männlichen Autoren (ECK, LÜBBE, MEIER-DALLACH, SOLARI, RIPPMANN, WIDMER, GOLOWIN) Überlegungen zur schweizerischen Identität und zum schweizerischen Heimatbegriff angestellt und weiterentwickelt. In der Einleitung zum Thema fragt ROGGEN, ob «der Schweizer/die Schweizerin ihr Land, das Land ihrer Väter und Mütter, überhaupt noch als Heimat» empfinden oder ob «die Qualität der Herkunft, des historisch-kulturellen, des geistigen und des biologischen Umfelds von sekundärem Interesse» sei, «in einer Zeit, in der die primären, existentiellen Probleme hierzulande gelöst scheinen und der Weg sich öffnet zur Wahrnehmung höherer Bedürfnisse, die sich in der Frage nach dem Sinn des Lebens und dem Wunsch nach 〈Selbstverwirklichung〉 manifestieren» (ROGGEN 1990: 9). Sorgen nicht gerade Publikationen dieser Art dafür, daß die von Roggen erwähnten Kategorien nicht von sekundärem Interesse bleiben? Das Beispiel «Störfall Heimat - Störfall Schweiz» soll illustrieren, daß der Heimatbegriff heutzutage eine zusätzliche Dimension erhalten hat. Nicht nur Politiker und Politikerinnen, auch Wissenschaftler und Wissenschaftlerinnen haben den Begriff für sich entdeckt. Es wird eine allgemeine Krise diagnostiziert, von einer bedrohten «Identität der Heimat» (von außen: zum Beispiel «Asylanten» und «Dealer»; von innen: zum Beispiel «Drogensüchtige» und «Linke») gesprochen, und davon werden psychosoziale Abwehrmechanismen im Menschen abgeleitet, indem behauptet wird, daß diffuse Identitätszustände zu Labilität und Panik tendieren. Damit lassen sich die in Krisenzeiten sich oft manifestierenden Erscheinungen wie Provinzialismus, Isolationismus, Rassismus usw. erklären. Doch was war zuerst: die attestierte Identitätskrise der Bevölkerung oder die sich in der Krise zeigenden Erscheinungen? Und wer leidet denn an einer Identitätskrise: die Herrschenden oder jene, welche diese als «das Volk» bezeichnen? Die Regression auf den Begriff Heimat erlaubt somit gleich zweierlei: Einerseits findet er häufig in politischen Reden Verwendung, andererseits eignet er sich auch vorzüglich für die Ursachenforschung, die mit ihm die festgestellten Krisensymptome zu erklären versucht.

Wobei die Erklärungen aufgrund einer zirkulären Argumentation eher dürftig bleiben, wie am Beispiel des konservativen Philosophen LÜBBE zu zeigen ist. Dieser greift auf den Begriff Heimat zurück, um das europaweite Anwachsen von Fremdenfeindlichkeit, Rechtsradikalismus oder Regionalismus zu erklären. Er konstatiert unter anderem eine zunehmende Bedrohung der Identität der Heimat, die die genannten Erscheinungen evoziert. Doch LÜBBE liefert eine nur auf den ersten Blick plausibel scheinende Erklärung für die Renaissance von Heimat. Er meint, daß wir der Herausforderung der Anpassung an sich dramatisch rasch verändernde Verhältnisse besser gewachsen seien, wenn wir uns durch 
Rückzugsmöglichkeiten in Sphären, wo sich gerade nichts ändert, Entlastung verschaffen. «Heimat, Herkunft, Region, Sprache, ja Konfession - diese kaum Änderungen unterworfenen Bestände werden in der modernen Welt Gewicht erhalten - aus Entlastungsgründen. Diese Prognose würde ich wagen» (LÜBBE 1990: 50). Das heißt, durch den Blick in die Vergangenheit würde der rasche gesellschaftliche Wandel zu bewältigen versucht. Plausibel könnte diese Erklärung sein, wenn es möglich wäre, die Geschwindigkeit des gesellschaftlichen Wandels zu messen. Weil das bisher aber noch niemandem gelungen ist, ist es auch unmöglich, zwischen Zeiten zu unterscheiden, in denen die genannten Kategorien wichtig sind, und Zeiten, in denen sie bedeutungslos sind.

Wenn ich noch mit LÜBBE dahingehend übereinstimme, $\mathrm{da}$ ß sich Leute mit dem Rückzug in die Vergangenheit Entlastung von den Herausforderungen der Gegenwart zu verschaffen versuchen, so halte ich es für höchst problematisch und unzulässig, wenn damit auch Erscheinungen wie Regionalismus, Rassismus und Faschismus erklärt werden. LÜBBES Erklärungsmodell ist ähnlich reduktionistisch und mechanistisch wie die ökonomische Erklärung des Nationalsozialismus. Rassismus ist ebensowenig wie Faschismus ein Problem der ökonomischen oder gesellschaftlichen Entwicklung. Ebensowenig wie die Leute automatisch zu Faschisten wurden, weil sie arbeitslos waren, werden die Leute nicht einfach zu Rassisten, weil sie mit dem gesellschaftlichen Wandel nicht mehr zu Rande kommen.

Nicht aus Entlastungsgründen erhalten Heimat, Herkunft, Region usw. in der modernen Welt Gewicht, sondern weil Leute mit Definitionsmacht diese Begriffe geschickt einzusetzen wissen, um daraus politisches Kapital zu schlagen. Dabei geht es um eine geschickte Ausnützung einer Angst. Es ist die Angst vor Multikultur, Massenkultur und Gegenkultur. Wenn Rechtspopulisten in ihren Reden auf Kategorien wie Heimat, Herkunft und Nation zurückgreifen, kann das einerseits von ihrer eigenen Angst zeugen, bei der Berührung mit dem Fremden zu zerfallen, andererseits aber auch vom Wissen darüber, welche heilende Wirkung diese Wörter bei bestimmten Leibern ausüben können. Heimat wird zur rettenden Vokabel gegen den alles verschlingenden Moloch Europa. Es geht dabei nicht um Demagogie, sondern um wirkliche Religion. Jene Menschen, die in ihrer Struktur bereits Elemente von faschistischem Denken und Fühlen aufweisen, sind relativ leicht für diese Religion zu gewinnen. Und zwar empfinden sie dieses Abgrenzungsbedürfnis nicht einfach deshalb, weil sie sich plötzlich von einer wirtschaftlichen und kulturellen Globalisierung bedroht fühlen, sondern weil sie grundsätzlich für ihre Existenz darauf angewiesen sind. Das Fremde und Andere empfinden sie als existentielle Bedrohung ihres ihnen selbst fremd bleibenden Körpers. Für ihre Selbstdefinierung sind sie auf außerhalb ihres Körpers hergestellte Identitätsmerkmale angewiesen. Solche Merkmale sind beispielsweise Nation, Rasse oder Ethnie. Wer sich für die Selbstidentität auf solche Dinge beruft, muß Angst haben, wie überhaupt das Bedürfnis, sich irgendwie dingfest machen zu wollen, auf Angst beruht. Der Erfolg von rechtskonservativen Politikern beruht auf der geschickten Ausnutzung dieser Angst.

\section{Heimat und Boden}

Hauptsächlich während der vor allem in Norddeutschland verbreiteten Heimatkunstbewegung um die Jahrhundertwende wurde das Heimatgefühl an jeweils einmalige und schicksalhafte Voraussetzungen von Landschaft und Stamm gebunden, womit deren Vorstellung von Heimat noch über den Territorialitätsbegriff der Verhaltensforschung hinausging. Während letztere nämlich für eine Spezies gewisse Minimalbedingungen in einer jeweils benötigten Fläche festmacht, diese aber als topographisch übertragbar ansieht, wurden bei der Heimatkunstbewegung die Bindungen als primäre zwischen jeweiliger Scholle und jeweiligem Menschen gesehen. Das heißt, jeder, der seinen Geburtsort verläßt und in eine noch so gleichartige Landschaft zieht, müßte, noch bevor Komponenten wie Sprache und soziale Beziehungen ins Spiel kommen, sich entheimatet fühlen (ROSSBACHER 1975).

Exemplarisch für die idealistische Grundposition der Verknüpfung von Mensch und äußerer Natur bzw. Boden möchte ich eine Abhandlung des in seiner Zeit bedeutenden Kulturphilosophen SPRANGER zitieren. An der Eröffnungsrede der «Studiengemeinschaft für wissenschaftliche Heimatkunde» im April 1923 sagte er: «Der Mensch hat, wo er auch lebe, immer eine Umwelt, ein für ihn und seinen Lebensvollzug bedeutsames «Milieu», nicht aber eine Heimat. Eine Heimat hat er nur da, wo er mit dem Boden und mit allem NaturhaftGeistigen, das diesem Boden entsprossen ist, innerlich verwachsen ist. (...) Von Heimat reden wir, wenn ein Fleck Erde betrachtet wird unter dem Gesichtspunkt seiner Totalbedeutung für die Erlebniswelt der dort lebenden Menschengruppe. Heimat ist erlebbare und erlebte Totalverbundenheit mit dem Boden. Und noch mehr: Heimat ist geistiges Wurzelgefühl» (SPRANGER 1973: 298).

Es ist unschwer, hier eine geistige Verwandtschaft zur Blut-und-Boden-Mystik des Nationalsozialismus auszumachen, mit deren Hilfe die faschistische Propaganda einen unauflöslichen Zusammenhang des Menschen mit einem ganz bestimmten geographischen Milieu zu konstruieren versuchte. Und die Nationalsozialisten haben bekanntlich solche Ideen aufgegriffen, um ihr Programm zu legitimieren und die Massen in ihrem Sinne zu mobilisieren. SPRANGER als geistigen Vater der Blut-und-Boden-Mystik zu bezeichnen oder sogar für deren fatale Folgen verantwortlich zu machen, wäre aber ebenso unzulässig wie zu behaupten, daß das Wetter von der Voraussage im Fernsehen abhängt. Der Faschismus hat weitaus komplexere Ursachen als das bloße Rezipieren faschistischer Ideen aus Büchern. ${ }^{3}$ Es wäre aber 
in der Tat mehr als naiv, wie HEINRICH in seiner eindrücklichen Dissertation zum Thema «Geographie und Faschismus» bemerkt, die politische Tendenz von Texten zwar anzuerkennen, die Übernahme ihrer Ideen in die gesellschaftliche Auseinandersetzung jedoch als vermeintlichen Mißbrauch zu bezeichnen (HEINRICH 1991: 389).

Liest man SPRANGERS Abhandlung heute, kommt sie einem übrigens nicht weniger antiquiert und beklemmend vor als gewisse Schriften aus heutiger Zeit. Wenn er etwa schreibt, daß ihm die Heimatkunde eine «Schule des Totalitätssinnes» ist, «die wir brauchen, um aus der geistigen Zerrissenheit der Gegenwart herauszukommen» (SPRANGER 1973: 317), dann sehen wir, wo LÜBBE seine geistigen Väter hat.

In der Schweiz war der prominenteste Vertreter der Position der Erdverwurzelung des Menschen der Zürcher Geograph EGLI. Durch seine ganzen Schriften zieht sich die Vorstellung der «menschlichen Erdbedingtheit». Im Extremfall spricht er auch schon mal von der "völkischen Gestaltungskraft des Schweizerbodens». Das «Schweizerblut» war seiner Ansicht nach ein Produkt von tektonisch-geologischen Faktoren, das heißt des Bodenprofils und der Landschaftsbeschaffenheit. Auf diese Vorstellung der Schweiz als Naturmonument griff auch die Schweiz in ihrer Suche nach einem neuen nationalen Selbstverständnis nach 1933 zurück, wie der Historiker TANNER darlegt. Damit verblieb zwar der helvetische Unabhängigkeitsdiskurs wie in Deutschland innerhalb der rassistischen «Blut-und-Boden-Mythologie», die Schweiz verlagerte ihr nationales Selbstverständnis jedoch vom Blut auf den Boden. Nicht dem Blut, sondern dem Raum wurde die einheitsstiftende Macht zugeschrieben. Neben den Literaten waren es insbesondere Geographen, ${ }^{4}$ die eine schicksalhafte Verbundenheit der Schweizer mit den Alpen propagierten. Für die staatspolitische Abgrenzungsrhetorik gegenüber Deutschland fand die Schweiz Ansatzpunkte im ideologischen Konstrukt des «Gotthardstaates». Aus dem harten Gotthardgranit entstiege ein kerngesunder Geist, aus welchem der schweizerische Staatsgedanke geboren wäre, wie die Landesregierung dies in einer 1938 veröffentlichten Kulturbotschaft erklärte. Dieses mit einer Ewigkeitsgarantie versehene nationale Selbstverständnis sah sich durch offene Grenzen und unkontrollierte Einwanderung gefährdet. Das politische Losungswort, welches sich gegenüber dem gesellschaftlichen Wandel lange Zeit ohne jegliche Abnützungserscheinungen halten sollte, war der «Kampf gegen die Überfremdung» (TANNER 1993: 16).

Noch 1976 vertrat EGLI die Ansicht, daß der Gotthard ohne Zweifel «für alle Schweizer ein gedankliches Zentrum des Lebensraumes», ein «landschaftliches und geistiges Element in der vaterländischen Ebene» (EGLI 1977: 15) sei. Überhaupt enthält diese Rede nochmals in geraffter Form beinahe alle Elemente seiner geodeterministischen Argumentationsweise, die EGLI seit den dreißiger Jahren immer wieder vorgetragen hatte. Ich zitiere lediglich einige Kernsätze dieser Rede, die EGLIS
Denkweise illustrieren sollen: «Unser Unbewußtes hingegen wird stark vom Raume mitgestaltet», «So also entsteht Heimat: durch das Wurzelfassen in einem landschaftlichen Raum», «Der Mensch wächst nicht nur in die Landschaft ein, die Landschaft dringt umgekehrt durch vielfältige Reizwirkungen ins Innere des Menschen vor» und der wahrscheinlich ungeheuerlichste Satz der ganzen Rede: «Die starken und reich variierten Umweltstimulanzien all der örtlichen und bildprägenden Psychotope wirken nun also auf Genpakete und setzen genetische Veranlagung in individuelle Wirklichkeit um.» Hier stützt sich EGLI auf die angeblich von der Biologie bewiesene Tatsache, daß die äußere Umwelt die Art und Weise bedinge, wie die genetische Veranlagung eines jeden Menschen in die individuelle Wirklichkeit umgesetzt wird, und will der nüchternen Wissenschaft für dieses glanzvolle Resultat beinahe «das Zugeständnis der 〈heiligen Nüchternheit〉 machen».

EGLI scheint sich hier von der trügerischen Plausibilität organischer Metaphern irrefuihren bzw. blenden zu lassen. Wie ARENDT konsequent aufgezeigt hat, ist diese Gefahr in der Diskussion der Rassenfrage besonders groß: «Jeder Rassismus, der weiße wie der schwarze, ist von Haus aus gewaltträchtig, weil er gegen natürliche, organische Gegebenheiten protestiert, eine schwarze oder eine weiße Haut, die nicht von Meinungen abhängen und an denen keine Macht etwas ändern könnte; kommt es hart auf hart, so bleibt nichts als die Ausrottung ihrer Träger. Rassismus ist, im Unterschied zur Rasse selbst, keine tatsächliche Gegebenheit, sondern eine zur Ideologie entartete Meinung, und die Taten, zu denen er führt, sind keine bloßen Reflexe, sondern Willensakte, die sich logisch aus gewissen pseudowissenschaftlichen Theorien ergeben» (ARENDT 1995: 75). Es sollte einsichtig geworden sein, daß es genau solche der Biologie entlehnten «pseudowissenschaftlichen Theorien» sind, die der Nährboden für viele -ismen sind, seien dies nun Rassismus, Regionalismus, Nationalismus oder Faschismus.

Auch wenn inzwischen die Bindungen an einen bestimmten Boden durch eine weltweite Globalisierung, verbunden mit einer praktisch uneingeschränkten Mobilität, mehr als widerlegt sein sollten, gibt es auch heute immer noch Leute, die Heimat am Herkunftsort festmachen bzw. die «Dämonien des Bodens» für die Raumgebundenheit des Menschen verantwortlich machen. Die Strategie der Moderne, den Raum zu homogenisieren, das heißt unsere Beziehungen $\mathrm{zu}$ ihm zu versachlichen, hat - wie jeder weiß - nicht gefruchtet. Homogene, freie Räume gibt es nicht. Wir leben in mit vielfachen, oft überschichteten Bedeutungen besetzten Räumen, in Räumen, die mit den unterschiedlichsten Qualitäten belegt sind. Wie wir angesichts aktueller regionalistischer und nationalistischer Tendenzen auf der ganzen Erde anerkennen müssen, ist die Aufklärung diesbezüglich offensichtlich noch nicht abgeschlossen. Es ist eben allem Anschein nach nicht so, daß sich Regionalismus und Moderne grundsätzlich ausschließen müssen. Im Gegenteil: War es nicht gerade die Moder- 
ne, die unser Verhältnis zum Raum erst emotionalisiert hatte? Heimat war bis ins 19. Jahrhundert in der Hauptsache kein emotional behafteter Begriff, sondern hatte vor allem rechtliche Bedeutung. Es überwog die rein sachliche Beschreibung eines bestimmten Raumbereichs. Der Regionalismus mag zwar bezogen auf die Ziele der Moderne als ein widersprüchliches Phänomen erscheinen, wurde aber erst in ihr überhaupt ins Leben gerufen. Ebenso dürfte Heimat als Folge der Aufklärung mythologisiert worden sein. Der Bedarf an Mythen kann als Antwort auf das rationale wissenschaftliche Begriffsdenken aufgefaßt werden, weil Mythen Aussagen und Vorstellungen über die Welt ermöglichen, die mehr diffus geahnt als reflektiert sind. Jeder Mythos befriedigt das Bedürfnis, der Realität eine andere Wirklichkeitsvorstellung entgegenzuhalten. Heimat als Mythos schlechthin läßt in einer versachlichten, geheimnislosen Welt sinnliche und emotionale Erfahrungen zu. In einer zunehmend aufgesplitterten Gesellschaft schafft es der Mythos, die Menschen zu versammeln und zu vereinigen. Deshalb sind Mythen gerade heute wieder so gefragt. Sterben die alten Mythen, greift das Chaos um sich, vor dem sich vor allem jene konservativen Kreise fürchten, deren größtes Bedürfnis Ordnung ist.

Das Verhältnis der Menschen zum Raum dürfte also vor der Moderne weitaus weniger bis gar nicht emotionalisiert gewesen sein, als es seither ist. Und obwohl die Gefühlswerte des Begriffs Heimat objektiv schon lange nicht mehr auf eine geographische Größe gerichtet sind, ist die subjektive Assoziation von Heimat mit geographischen Räumen und Regionen allgegenwärtig. Trotz oder gerade wegen der fortschreitenden «Entzauberung der Welt» (WEBER) ist unser Verhältnis zum Raum nicht gleichgültig geworden. Es bleibt antimodern. Und mit antimodernen Inhalten läßt sich nach wie vor erfolgreich Politik machen.

\section{Alternativen zur Rettung eines schönen Wortes}

Am konsequentesten und radikalsten ist aus handlungstheoretischer Position eine nichtraumzentrierte Sicht der sozial-kulturellen Wirklichkeitsdimensionen gefordert worden (vgl. vor allem WERLEN 1993a, 1993b, 1995, 1997). Da Raum weder ein Gegenstand noch ein empirischer Begriff ist, sollte man ihn auch nicht weiter als besonderen Forschungsgegenstand der Geographie betrachten. Anstelle von Raum schlagen Handlungstheoretiker das menschliche Handeln als Forschungseinheit vor. Unter einem handlungszentrierten Gesichtspunkt ist auch Heimat lediglich ein formal-klassifikatorischer Begriff. Formal, weil er sich nicht auf inhaltliche Merkmale von materiellen Gegebenheiten bezieht, und klassifikatorisch, weil er Ordnungsbeschreibungen von materiellen Objekten und die Orientierung in der physischen Welt zuläßt. Es gibt keine räumlichen Heimaten. Und Wissenschafter sollten sich nicht - wie dies HARD auf eindrückliche Weise schon vor Jahren für die Wörter «Landschaft» und «landschaftlich» gezeigt hat
(HARD 1970) - allein aufgrund der Tatsache, daß das Wort Heimat existiert, sowie des Glaubens, daß einem Substantiv doch auch ein real existierendes Objekt entsprechen müsse, zur Behauptung versteigen, daß Heimat «ein konkretes Forschungsobjekt» sei. Räume und Landschaften an sich sind nichts. Sie haben aber durchaus symbolische Bedeutung. Denn die Landschaften und ihre Elemente werden von den Menschen symbolisch aufgeladen, womit ihnen gleichzeitig eine hervorrufende Kraft zugeschrieben wird. Ohne diese Besetzung wären sie nichts. Eine Bedeutung ist kein Ding. Jedes vermeintlich an einen Raum gebundene Heimaterlebnis knüpft sich in erster Linie an die Menschen, die eine Landschaft bzw. einen Raum erst zur Heimat machen. Vor allem die Geographie sollte sich dem sozialen Phänomen Heimat nicht weiter auf dem Umweg über den Raum nähern. Von der Heimat als bodenverhafteter Lebensmitte sollte definitiv Abschied genommen werden. Nur wenn Heimat gegenüber dem Fremden und Andersartigen offen ist, läßt sich auf redliche Weise mit Menschen aus verschiedenen Nationen zusammenleben. Heimat verlangt zudem, wenn sie mehr als nur Regionalismus sein will, daß wir die Umgebung, das Regionale zu einer von uns selbst bewerteten Umwelt machen.

Dies ist auch die Ansicht von DAUM, Dozent an der Universität Hannover im Fachbereich Erziehungswissenschaften, der sich mit der Frage nach neuen Inhalten für Heimat beschäftigt (DAUM 1990). Ihm geht es vor allem um die Bestimmung der aktuellen Bedingungen, unter denen Kinder und Jugendliche heute aufwachsen. DAUM distanziert sich deutlich von einer rückwärtsgewandten Heimatkunde und plädiert statt dessen für ein aktiv die Umwelt aneignendes und verarbeitendes Konzept von Heimat. Sein zukunftsorientiertes Heimatverständnis orientiert sich stark an der auf eine bessere Zukunft gerichteten Utopie im Sinne BLOCHS. "Heimat entsteht dort, wo wir Verhaltenssicherheit erfahren, und zwar im Umgang mit den Dingen, Verhältnissen und Menschen. Hieraus erwachsen Vertrautheit und Überschaubarkeit, jedoch nicht im Sinne einer statischen Ordnung. Gemeint ist vielmehr eine soziale Kompetenz, die Dinge, Verhältnisse und Personen zu beeinflussen und mitzugestalten sowie sich selbst als Subjekt darin wiederzuerkennen.» Bei diesem aktiv verstandenen Heimatbegriff geht es insbesondere um «die Wiedergewinnung der sinnlichen Erfahrbarkeit». In ihrer Symbolträchtigkeit repräsentiert Heimat also die Vertrautheit, Nähe und Verläßlichkeit von konkreten Lebensverhältnissen. Nach DAUM kann Heimat durchaus mit Sinn und Verstand gefüllt werden, darum will er den Begriff auch nicht aufgeben oder nach begrifflichen Alternativen suchen (etwa "Territorialität»). Denn das hieße, das Feld allein den ewig Vorgestrigen zu überlassen. Ein triftiges Argument, sich für die Utopie Heimat einzusetzen und neue Inhalte für den Begriff zu suchen. 


\section{Kommt bald das Ende einer räumlich verstandenen Heimat?}

Es ist nun aber durchaus denkbar, daß sich alle Bemühungen für einen aufgeklärten Diskurs bezüglich Raum in absehbarer Zeit als vergeblich herausstellen werden. War alle Mühe umsonst? Wird der Raum ebenso von neuen Technologien abgeschafft, wie auch zunehmend die Zeit abgeschafft wird? Wie er ja auch im 14./15. Jahrhundert einmal von raumgreifenden Italienern konstruiert wurde. Folgende These von THEWELEIT halte ich für durchaus plausibel: «Das Licht im Kopf unserer (?) Kinder wird sich vom Raum, von Lichtquellen und von Geschichte gelöst haben: Bildschirme, Monitoren transformieren Licht in ein flächiges Flimmern ohne Lichtquelle. Nach der Abschaffung der Zeit wird die Abschaffung des Raums hergestellt von augenblicklichen Technologien» (THEWELEIT 1988: 462). Als ausreichend evidentes Beispiel fügt THEWELEIT hin$\mathrm{zu}$, daß der Weltraum momentan ausschließlich auf Monitoren spielt, in einem antiräumlichen Medium. In der Tat verlieren die klassischen Raumerfahrungen der großstädtischen Zivilisation ihre Konsistenz. «Die urbanen Geographien sind im Begriff, sich aufzulösen», wie KREIMEIER schreibt. Zwar durchmessen wir immer noch Räume, um von einem Ort zum nächsten zu kommen, doch mit der «primären Realität» vor unserer Haustür konkurrieren zunehmend «die vielfältigen Realitäten der elektronischen Vernetzung - ein Kontinent indirekter Bilder und Zeichen, der genauer als Archipel zu beschreiben wäre, als verzweigtes System, das mit seinen Apparaturen, 〈Interfaces〉 und variierenden Botschaften unseren Alltag durchdringt» (KREIMEIER 1995: 16). Das Fernsehen produziert neue Wirklichkeiten. Der traditionelle urbane Raum jedoch verliert immer mehr an «Realität». Die urbanen Geographien werden zunehmend von medialen Geographien überlagert und ersetzt.

Die These vom verschwindenden Raum hat der bekannte Medienforscher Marshall McLUHAN schon 1967 aufgestellt. McLUHAN: "Wir leben in einer brandneuen Welt der Gleichzeitigkeit. Die 〈Zeit〉 hat aufgehört, der 〈Raum〉 ist dahingeschwunden. Wir leben heute in einem globalen Dorf... in einem gleichzeitigen Happening» (McLUHAN 1969: 63). Auch der Leiter des aktuellen McLUHAN-Programms über Kultur und Technologie an der Fakultät für Informationsstudien der Universität von Toronto, DE KERCKHOVE, vertritt die These, daß telematische Netze Raum und Zeit abschaffen. Er hält jedoch die Vorstellung vom «globalen Dorf» ungeeignet zur Beschreibung der neuen Bedingungen vernetzter Kommunikation. «Während das Fernsehen einen gemeinsamen Raum schuf und diesen auch wahrnehmbar machte, setzen Datennetze die Dimensionen von Raum und Zeit außer Kraft und damit auch die traditionellen Bezugspunkte der Identität, und zwar sowohl der persönlichen als auch der kollektiven» (DE KERCKHOVE 1995).
Ob diese Prognosen jedoch tatsächlich eintreten, kann zurzeit freilich noch nicht gesagt werden. Momentan deutet leider vieles darauf hin, daß LÜBBE mit seiner These, daß die kaum sich verändernden Bestände wie zum Beispiel Heimat und Region wieder zunehmend Gewicht erhalten werden, doch recht bekommen wird. Womit auch die Unheimlichkeit von Heimat bis auf weiteres erhalten bliebe.

\section{Anmerkungen}

1 Der Beitrag ist ein stark überarbeiteter und erweiterter Vortrag mit dem Titel "Über die Unheimlichkeit von Heimat", den ich am ASG-Symposium "Regionalismus" der SANW-Jahresversammlung vom 8. September 1995 an der Hochschule St. Gallen gehalten habe. Er besteht aus Auszügen des theoretischen Teils meiner Dissertation "Vermeintliche Heimaten. Über den Bedeutungswandel von Heimat in der Postmoderne am Beispiel einer Untersuchung über Schweizer Rentnerinnen und Rentner an der Costa Blanca" (im Druck).

2 Man kann die Unheimlichkeit von Heimat aber auch dialektisch umkehren. Denn das Empfinden, daß man tatsächlich in ganz bestimmten Räumen und Zeiten zu kleben scheint, aus denen man sich sein Leben lang loszulösen versucht, ist nicht anders als unheimlich zu bezeichnen. Wie sonst läßt sich die weitverbreitete Faszination von Sciencefiction-Filmen und -Büchern erklären, die neben anderen Gründen vor allem darin besteht, sich aus Raum und Zeit "wegbeamen" zu können?

3 Die überzeugendste Faschismustheorie hat meines Erachtens Klaus THEWELEIT in seinem Buch Männerphantasien geliefert (THEWELEIT 1986). Man wird darin auch Antworten auf die Fragen finden, warum welche Leute aus welchen Gründen auf die von LÜBBE genannten Kategorien ansprechen. Man kann diese als übergestülpte Identitätsangebote zur Erhaltung von nicht zu Ende geborenen Körpern ansehen.

4 Ich verwende hier absichtlich nur die männliche Form, da mir keine Frauen bekannt sind, die diese These in jener Zeit öffentlich vertraten.

\section{Literatur}

ARENDT, H. (1995): Macht und Gewalt. München: Piper (erstmals New York: Harcourt, Brace \& World, Inc. 1970), 8. Auflage.

BARTHES, R. (1964): Mythen des Alltags. Frankfurt am Main: Suhrkamp (erstmals Paris: Editions du Seuil 1957).

DAUM, E. (1990): Was heißt hier Heimat? Neue Inhalte für einen emotional befrachteten Begriff. In: NZZ, Nr. 214, 15./16. September, S. 25.

DE KERCKHOVE, D. (1995): Vom globalen Dorf zum globalen Denken. In: Unesco-Kurier, 36. Jg., Nr. 2, S. 16-18.

EGLI, E. (1977): Geborgenheit im Raum. Zum Begriff der Heimat. Vortrag vom 15. Mai 1976 an der 5. Jahresversammlung der Stiftung für abendländische Besinnung im Zunfthaus am Neumarkt, Zürich. Schaffhausen: Novalis. 
HARD, G. (1970): Die "Landschaft" der Sprache und die "Landschaft" der Geographen. Semantische und forschungslogische Studien. Bonn: Ferd. Dümmlers Verlag (= Colloquium Geographicum 11).

HEINRICH, H. A. (1991): Politische Affinität zwischen geographischer Forschung und dem Faschismus im Spiegel der Fachzeitschriften. Ein Beitrag zur Geschichte der Geographie in Deutschland von 1920 bis 1945. Gießen: Selbstverlag des Geographischen Instituts der Justus-LiebigUniversität Gießen (= Gießener Geographische Schriften Heft 70)

KOS, W. (1993): Das Volkslied im leeren Raum. Eine Begriffsbestimmung. In: «Du», Nr. 7, Juli, S. 42-45.

KREIMEIER, K. (1995): Lob des Fernsehens. München: Hanser Verlag.

LÜBBE, H., u. ECK, C. D. (1990): Diskussion 1. In: Institut für Angewandte Psychologie IAP Zürich (Hrsg.) (1990): Störfall Heimat - Störfall Schweiz. Anmerkungen zum schweizerischen Selbstverständnis im Jahre 699 nach Rütli und im Jahre 2 vor Europa. Jahrestagung 1990. Baden: Kommissionsverlag Baden-Verlag, S. 40-50.

MCLUHAN, M., QUENTIN, F., U. JEROME, A. (1969): Das Medium ist Massage. Frankfurt am Main; Berlin: Ullstein.

ROGGEN, B. (1990): Zum Thema. In: Institut für Angewandte Psychologie IAP Zürich (Hrsg.) (1990): S. 9-10.

ROSSBACHER, K. (1975): Heimatkunstbewegung und Heimatroman. Zu einer Literatursoziologie der Jahrhundertwende. Stuttgart: Ernst Klett Verlag, 1 (= Literaturwissenschaft Gesellschaftswissenschaft 13).

SPRANGER, E. (1973): Der Bildungswert der Heimatkunde. In: BOLLNOW, O. F., u. BRÄUER G. (Hrsg.): Eduard Spran- ger: Gesammelte Schriften. Bd. 2: Philosophische Pädagogik. Tübingen: Max Niemeyer Verlag, S. 294-319.

TANNER, J. (1993): Geist aus Gotthardgranit. In: WoZ, Nr. 48, 3. Dezember, S. 16.

THEWELEIT, K. (1986): Männerphantasien. Bd. 1: Frauen, Fluten, Körper, Geschichte. Bd. 2: Männerkörper - zur Psychoanalyse des Weißen Terrors. Basel; Frankfurt am Main: Stroemfeld/Roter Stern.

THEWELEIT, K. (1988): Buch der Könige. Bd. 1: Orpheus und Eurydike. Frankfurt am Main: Stroemfeld/Roter Stern.

THEWELEIT, K. (1989): Three Steps aus 31 Jahren mit Miles. In: "Du», Nr. 8, August, S. 82-84.

VORLÄNDER, H. (1984): Heimat und Heimaterziehung im Nationalsozialismus. In: KNOCH, P., u. LEEB, T. (Hrsg.): Heimat oder Region? Grundzüge einer Didaktik der Regionalgeschichte. Frankfurt am Main: Diesterweg, S. 30-43.

WERLEN, B. (1993a): Gibt es eine Geographie ohne Raum? Zum Verhältnis von traditioneller Geographie und zeitgenössischen Gesellschaften. In: Erdkunde, Bd. 47, Nr. 4, S. 241255.

WERLEN, B. (1993b): Identität und Raum. Regionalismus und Nationalismus. In: "Soziographie", 6. Jg., Nr. 7, S. 39-73.

WERLEN, B. (1995): Sozialgeographie alltäglicher Regionalisierungen. Bd. 1: Zur Ontologie von Gesellschaft und Raum (= Erdkundliches Wissen 116), Stuttgart: Franz Steiner Verlag.

WERLEN, B. (1997): Raum, Körper und Identität. Traditionelle Denkfiguren in sozialgeographischer Reinterpretation. In: STEINER, D. (Hrsg.): Mensch und Lebensraum. Fragen zu Identität und Wissen. Opladen: Westdeutscher Verlag, S. $147-168$.

\title{
Alfred Bögli
}

\begin{abstract}
Am 12. Februar 1998 ist Prof. Dr. Alfred Bögli in seinem 86. Altersjahr verstorben.
Ein Nachruf wird in der nächsten Nummer der Geogra- phica Helvetica folgen.

Die Redaktion 